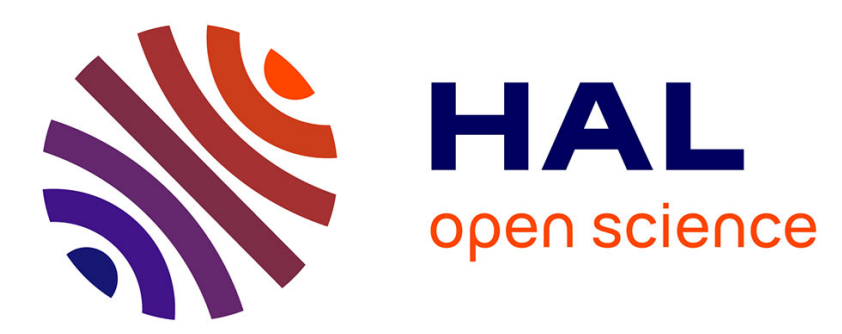

\title{
Stable Perfectly Matched Layers with Lorentz transformation for the convected Helmholtz equation
}

Philippe Marchner, Hadrien Beriot, Xavier Antoine, Christophe Geuzaine

\section{To cite this version:}

Philippe Marchner, Hadrien Beriot, Xavier Antoine, Christophe Geuzaine. Stable Perfectly Matched Layers with Lorentz transformation for the convected Helmholtz equation. Journal of Computational Physics, 2021, 10.1016/j.jcp.2021.110180 . hal-02556182v2

\section{HAL Id: hal-02556182 \\ https://hal.science/hal-02556182v2}

Submitted on 10 Feb 2021

HAL is a multi-disciplinary open access archive for the deposit and dissemination of scientific research documents, whether they are published or not. The documents may come from teaching and research institutions in France or abroad, or from public or private research centers.
L'archive ouverte pluridisciplinaire HAL, est destinée au dépôt et à la diffusion de documents scientifiques de niveau recherche, publiés ou non, émanant des établissements d'enseignement et de recherche français ou étrangers, des laboratoires publics ou privés. 


\title{
Stable Perfectly Matched Layers with Lorentz transformation for the convected Helmholtz equation
}

\author{
Philippe Marchner ${ }^{\mathrm{a}, \mathrm{b}, \mathrm{c}}$, Hadrien Bériot ${ }^{\mathrm{a}}$, Xavier Antoine ${ }^{\mathrm{b}}$, Christophe Geuzaine ${ }^{\mathrm{c}}$ \\ ${ }^{a}$ Siemens Industry Software SAS, 150 Avenue de la République, 92320 Châtillon, France \\ ${ }^{b}$ Université de Lorraine, CNRS, Inria, IECL, F-54000 Nancy, France \\ ${ }^{c}$ Université de Liège, Institut Montefiore B28, 4000 Liège, Belgium
}

\begin{abstract}
Perfectly Matched Layers (PMLs) appear as a popular alternative to non-reflecting boundary conditions for wave-type problems. The core idea is to extend the computational domain by a fictitious layer with specific absorption properties such that the wave amplitude decays significantly and does not produce back reflections. In the context of convected acoustics, it is well-known that PMLs are exposed to stability issues in the frequency and time domain. It is caused by a mismatch between the phase velocity on which the PML acts, and the group velocity which carries the energy of the wave. The objective of this study is to take advantage of the Lorentz transformation in order to design stable perfectly matched layers for generally shaped convex domains in a uniform mean flow of arbitrary orientation. We aim at presenting a pedagogical approach to tackle the stability issue. The robustness of the approach is also demonstrated through several two-dimensional high-order finite element simulations of increasing complexity.
\end{abstract}

Keywords: Perfectly matched layers, stability, Lorentz transformation, convected Helmholtz equation, cross flow, high-order FEM

\section{Introduction}

The Perfectly Matched Layer technique has been introduced by Bérenger for electromagnetics in 1994 [1]. By virtue of its versatility and simplicity, it has gained a large popularity as an alternative to non-reflecting boundary conditions for a wide range of wave-type problems. Further developments have led to a general derivation based on a complex coordinate stretching, in which the PML is viewed as an analytic continuation of the wave equation $[2,3]$. The complex stretching approach has been theoretically analyzed and extended to curvilinear coordinates by Collino and Monk [4]. While the PML is well-understood on the continuous level, its performance on a discrete level is hard to infer a priori and often cumbersome to optimize [5, 6]. Bermúdez et al. [7] introduced an unbounded stretching function which turns out to be more robust with respect to the PML parameters for finite element Helmholtz problems. The authors showed that this peculiar function allows to exactly recover the solution of the acoustic scattering problem [8]. A comprehensive and concise introduction to the PML developments can be found in [9].

The issue of the PML stability for flow acoustics application has first been raised for the linearized Euler equations in the time domain $[10,11,12]$. It has been shown that the convection may generate a sign mismatch between the phase velocity on which the PML acts, and the group velocity carrying the energy of the wave, which in turn leads to an exponentially growing solution inside the layer. General, theoretical results have been obtained in [13]. Hu proposed a stable formulation in unsplit physical variables [14] and later extended it to non-uniform flows [15]. Bécache et al. analyzed a closely related formulation for time-harmonic applications [16] and mathematically justified the associated convergence properties. Stability issues have mainly been addressed for uni-axial flows, and/or for Cartesian PMLs with axis-aligned flows. There are fewer works examining the issue of PML stability in uniform flows of arbitrary orientation. Hagstrom and

Email addresses: philippe.marchner@siemens.com (Philippe Marchner), hadrien.beriot@siemens.com (Hadrien Bériot), xavier.antoine@univ-lorraine.fr (Xavier Antoine), cgeuzaine@uliege.be (Christophe Geuzaine) 
Nazarov [17, 18] and Nataf [19] proposed novel methodologies to include cross flows for the linearized Euler equations. Dubois et al. [20] used the Lorentz transformation to design a stable absorbing layer. Diaz and Joly [21] analyzed the effect of a similar transformation based on the slowness curve properties, in the spirit of [22] for anisotropic media. Parrish and $\mathrm{Hu}$ [23] derived the $x, y$ and corner layers independently. Most of these works are devoted to the linearized Euler equations in the time domain, and the available literature on the stabilization of PMLs for the convected Helmholtz equation in cross flows is still scarce. This is however of practical interest, since the convected Helmholtz operator forms the basis of several finite element codes which are routinely used in industry, e.g. to support the acoustic design of turbofan engines [24, 25]. While it may be argued that stability issues are less problematic in the frequency domain, they significantly impact the numerical accuracy, especially at high Mach number flows, as will be demonstrated in this study.

The underlying idea behind a stable formulation relies on the possibility of applying a transformation to the wave equation that removes the presence of the so-called 'backward' or 'inverse' waves. Within the framework of convected acoustics, similarity transformations have been studied by Amiet and Sears [26], Taylor [27] and later by Chapman [28]. The Lorentz transformation, that is commonly used in special relativity [29], seems to emerge as a general tool for similarity transformations. Theoretical works have for instance been carried out using geometric algebra in [30, 31]. The applications of such a transformation in acoustics has been recently reviewed by $\mathrm{Hu}$ et al. [32], where the transformation is referred to as 'PrandtlGlauert-Lorentz', to highlight the different historical contributions. It has also been successfully used in the context of boundary element methods, where it allows to account for the convection, while still resorting to the conventional Green's function kernel [33, 34].

In this work, we focus on the Lorentz transformation to design general stable convex PML domains for the convected wave equation in the frequency domain. We aim at providing a clear derivation with illustrative examples, as well as an implementation strategy for practical purposes. The paper is organized as follows: we first recall the convected wave equation and introduce the Lorentz transformation, the PML stretching and carry out a plane wave stability analysis. In a second part, the stability of PMLs for duct propagation in a uniaxial flow is revisited. The third part examines the stabilization in free field for a two-dimensional Cartesian PML. Finally, stable PMLs are introduced for generally shaped convex PML domains in the presence of a general uniform cross flow. Numerical examples are provided throughout the study in order to illustrate the efficiency of the stabilized formulations.

\section{Lorentz transformation, PML and stability analysis}

We focus in this paper on the convected wave equation. It is a scalar equation for the acoustic potential $\varphi$ that describes the propagation of acoustic perturbations in a moving flow. It writes

$$
\mathcal{L} \varphi(\boldsymbol{x}, t)=\left\{-\frac{1}{c_{0}^{2}}\left(\partial_{t}+\boldsymbol{v}_{\mathbf{0}} \cdot \nabla_{\boldsymbol{x}}\right)^{2}+\Delta_{\boldsymbol{x}}\right\} \varphi(\boldsymbol{x}, t)=-f(\boldsymbol{x}, t),
$$

where $\boldsymbol{x}=(x, y)$ is the spatial variable, $t$ the time variable, $c_{0}$ the local speed of sound, $\boldsymbol{v}_{0}=\left(v_{x}, v_{y}\right)^{T}$ the local mean flow velocity vector and $f$ denotes the distribution of volume sources that are assumed to be compactly supported. The notations $\nabla_{\boldsymbol{x}}$ and $\Delta_{\boldsymbol{x}}$ respectively denote the spatial gradient and Laplacian operators. We introduce the local Mach number $M=\left\|\boldsymbol{v}_{0}\right\| / c_{0}$, which is the ratio between the local mean flow velocity to the speed of sound. The mean flow is assumed to be uniform and subsonic. It implies that the mean flow components are constant and that the condition $M<1$ holds. The speed of sound is as well assumed constant. The differential operator $\mathcal{L}$, referred to as convected Helmholtz operator, can be expanded as

$$
\mathcal{L}=\left(1-\frac{v_{x}^{2}}{c_{0}^{2}}\right) \partial_{x}^{2}+\left(1-\frac{v_{y}^{2}}{c_{0}^{2}}\right) \partial_{y}^{2}-2 \frac{v_{x} v_{y}}{c_{0}^{2}} \partial_{x y}^{2}-2 \frac{v_{x}}{c_{0}^{2}} \partial_{t x}^{2}-2 \frac{v_{y}}{c_{0}^{2}} \partial_{t y}^{2}-\frac{1}{c_{0}^{2}} \partial_{t}^{2} .
$$

In the frequency domain, we use the $e^{i \omega t}$ convention and readily get

$$
\mathcal{L}=\left(1-M_{x}^{2}\right) \partial_{x}^{2}+\left(1-M_{y}^{2}\right) \partial_{y}^{2}-2 M_{x} M_{y} \partial_{x y}^{2}-2 i k_{0} M_{x} \partial_{x}-2 i k_{0} M_{y} \partial_{y}+k_{0}^{2},
$$


where we have denoted $M_{x}=v_{x} / c_{0}, M_{y}=v_{y} / c_{0}$ the mean flow components, $\omega$ the angular frequency and $k_{0}=\omega / c_{0}$ the free field wavenumber. Note that the acoustic pressure and velocity fluctuations can be recovered from the acoustic potential by the relations

$$
\mathrm{p}(\boldsymbol{x}, t)=-\rho_{0}\left(\partial_{t}+\boldsymbol{v}_{0} \cdot \nabla\right) \varphi(\boldsymbol{x}, t), \quad \mathbf{v}(\boldsymbol{x}, t)=\nabla \varphi(\boldsymbol{x}, t),
$$

where $\rho_{0}$ is the fluid density.

\subsection{Lorentz transformation}

Various transformations are used in the literature for tackling sound propagation in uniform flow, such as Doppler factors, Lorentz transformations or Prandtl-Glauert coordinates. Motivated by the puzzling observation that these transformations are sometimes inconsistent, Chapman [28] has proposed a framework for these similarity variables. It consists in observing a given field $\varphi$ (here an acoustic perturbation), with a different space and time representation $(x, t) \rightarrow\left(x^{\prime}, t^{\prime}\right)$, yielding a different mathematical function $\varphi^{\prime}$

$$
\varphi^{\prime}\left(\boldsymbol{x}^{\prime}, t^{\prime}\right)=\varphi(\boldsymbol{x}, t) \text {. }
$$

The underlying idea is to modify the solution of the convected Helmholtz equation such that $\varphi^{\prime}$ is solution of a classical wave equation with modified, effective properties.

$\mathrm{Hu}$ et al. [32] emphasized that such a representation is not unique, which might explain the development of various methods. One solution arises from the Lorentz transformation in the theory of special relativity [29, chapter 11]. It turns out that there is a remarkable geometric link between flow acoustics and relativity, where acoustic waves are seen as fluctuations in a Lorentzian geometry [35]. For example, the connection is useful to build an analogue model for black holes and observe their properties as a fluid [36], the speed of sound playing the role of the speed of light. This analogy has been clarified and formalized with tools from geometry algebra [30]. For our purposes, the relevant representation comes through a combination of a Lorentz and Galilean transformation. It writes, in its vectorial form

$$
t^{\prime}=\beta t+\frac{(\boldsymbol{M} \cdot \boldsymbol{x})}{\beta c_{0}}, \quad \boldsymbol{x}^{\prime}=\boldsymbol{x}+\frac{(\boldsymbol{M} \cdot \boldsymbol{x})}{\beta(1+\beta)} \boldsymbol{M}, \quad \beta=\sqrt{1-M^{2}}, \quad \boldsymbol{M}=\left(\begin{array}{l}
M_{x} \\
M_{y}
\end{array}\right),
$$

where $\beta$ can be seen as a frequency factor. The modified spatial coordinates are also known as generalized Prandtl-Glauert coordinates, which are used to express compressibility effects for steady subsonic flows [37]. We choose to call transformation (2.4) the Lorentz transformation. We would like to mention that the transformation might be written through hyperbolic rotations and more generally rotors [31]. The partial derivatives can be computed thanks to the chain differentiation rule. In two-dimensions, one obtains

$$
\begin{aligned}
\frac{\partial}{\partial x} & =\left(1+\frac{M_{x}^{2}}{\beta(1+\beta)}\right) \frac{\partial}{\partial x^{\prime}}+\frac{M_{x} M_{y}}{\beta(1+\beta)} \frac{\partial}{\partial y^{\prime}}+\frac{M_{x}}{\beta c_{0}} \frac{\partial}{\partial t^{\prime}}, \\
\frac{\partial}{\partial y} & =\frac{M_{x} M_{y}}{\beta(1+\beta)} \frac{\partial}{\partial x^{\prime}}+\left(1+\frac{M_{y}^{2}}{\beta(1+\beta)}\right) \frac{\partial}{\partial y^{\prime}}+\frac{M_{y}}{\beta c_{0}} \frac{\partial}{\partial t^{\prime}}, \\
\frac{\partial}{\partial t} & =\beta \frac{\partial}{\partial t^{\prime}} .
\end{aligned}
$$

The extension to the three-dimensional case is straightforward, but we will restrict the analysis in twodimensions for conciseness. In the frequency domain, the field $\varphi(x, \omega)$ may be developed through its Fourier transform as

$$
\varphi(\boldsymbol{x}, \omega)=\int_{-\infty}^{+\infty} \varphi(\boldsymbol{x}, t) e^{-i \omega t} d t
$$

and using the introduced transformation (2.4) leads to

$$
\varphi(\boldsymbol{x}, \omega)=\int_{-\infty}^{+\infty} \varphi^{\prime}\left(\boldsymbol{x}^{\prime}, t^{\prime}\right) e^{-i \omega\left(\frac{t^{\prime}}{\beta}-\frac{(M \cdot \boldsymbol{x})}{\beta^{2} c_{0}}\right)} \frac{d t^{\prime}}{\beta} .
$$


We may define $\omega^{\prime}=\frac{\omega}{\beta}$ and obtain the frequency domain relation

$$
\varphi(\boldsymbol{x}, \omega)=\frac{1}{\beta} \varphi^{\prime}\left(\boldsymbol{x}^{\prime}, \omega^{\prime}\right) e^{i k_{0}^{\prime}\left(\boldsymbol{M} \cdot \boldsymbol{x}^{\prime}\right)}, \quad k_{0}^{\prime}=\frac{\omega^{\prime}}{c_{0}} .
$$

It follows that a translation and contraction in the time domain correspond respectively to a phase shift and scaling factor in the frequency domain. Thanks to the Lorentz transformation, the convected Helmholtz equation exactly reduces to a Helmholtz equation with a modified free field wavenumber and right-hand side

$$
\mathcal{L}^{\prime} \varphi^{\prime}\left(\boldsymbol{x}^{\prime}, \omega^{\prime}\right)=\left\{\partial_{x^{\prime}}^{2}+\partial_{y^{\prime}}^{2}+k_{0}^{\prime 2}\right\} \varphi^{\prime}\left(\boldsymbol{x}^{\prime}, \omega^{\prime}\right)=-f^{\prime}\left(\boldsymbol{x}^{\prime}, \omega^{\prime}\right) .
$$

We recall that this transformation is valid for a uniform mean flow of arbitrary direction, in time or frequency domain.

\subsection{PML as a complex stretching}

Let us consider a one-dimensional right half-space and truncate the unbounded domain by the open strip $\Omega=\left[0, L_{\mathrm{PML}}\right)$, called the global domain. We introduce the physical domain $\Omega_{\mathrm{phy}}=[0, L)$ and the PML domain $\Omega_{\mathrm{PML}}=\left(L, L_{\mathrm{PML}}\right)$. They share a common boundary at $x=L$ which is denoted $\Gamma_{\mathrm{int}}$. At the continuous level, the PML in a given direction can be seen as a stretch of the spatial coordinate in the complex space by a transformation of the form

$$
\widetilde{x}(x, \omega)=x-\frac{i}{k_{0}} \zeta(x), \quad \zeta(x)=\int_{L}^{x} \sigma(s) d s, \quad x \in\left(L, L_{\mathrm{PML}}\right),
$$

and the derivative writes

$$
\partial_{\widetilde{x}}=\left(1-\frac{i}{k_{0}} \sigma(x)\right) \partial_{x}=\gamma_{x} \partial_{x},
$$

where $\sigma(x)>0$ is called the absorbing function or absorbing profile. The choice of $\sigma$ is fundamental to ensure the quality of the PML. It is assumed to be positive, smooth and increasing in $\Omega_{\mathrm{PML}}$ and set to zero elsewhere.

In all that follows, we will use Bermúdez et al.'s unbounded function [7]

$$
\zeta(x)=-\sigma_{0} \ln \left(\frac{L_{\mathrm{PML}}-x}{L_{\mathrm{PML}}-L}\right), \quad \sigma(x)=\frac{\sigma_{0}}{L_{\mathrm{PML}}-x}, \quad x \in\left(L, L_{\mathrm{PML}}\right), \quad \sigma_{0} \in \mathbb{C},
$$

where $\sigma_{0}$ is a parameter to be selected. It is chosen to be purely real for propagative waves and complex for evanescent waves. Let us consider a plane wave traveling along the positive $x$-axis with wavenumber $k_{x}$. In $\Omega_{\mathrm{PML}}$, the plane wave becomes

$$
\widetilde{\varphi}(x, \omega)=e^{-i k_{x} \widetilde{x}}=e^{-i k_{x} x} e^{-\frac{k_{x}}{k_{0}} \zeta(x)}, \quad x \in\left(L, L_{\mathrm{PML}}\right),
$$

and the use of the unbounded function results in

$$
\widetilde{\varphi}(x, \omega)=e^{-i k_{x} x}\left(\frac{L_{\mathrm{PML}}-x}{L_{\mathrm{PML}}-L}\right)^{\sigma_{0} \frac{k_{x}}{k_{0}}}, \quad x \in\left(L, L_{\mathrm{PML}}\right) .
$$

Since $\widetilde{\varphi}$ is zero at the end of the layer, the wave is perfectly absorbed on the continuous level. If we further choose $\sigma_{0}=k_{0} / k_{x}$, that is the phase velocity of the wave, we get a linear decay in the PML. From a discrete, finite element point of view, it means that a single linear element can totally cancel out reflections. In practical computations however, there is no a priori knowledge on the phase velocity of the wave hitting the PML interface. For example, as soon as the incidence angle of the wave $\theta_{\mathrm{w}}$ is non-zero the propagation constant becomes $k_{x}=k_{0} \cos \left(\theta_{\mathrm{w}}\right)$. While Bermúdez et al.'s function might lead to an 'exact' PML, the decaying function is often not linear nor polynomial, thus leading to interpolation errors by the numerical scheme. In convected acoustics, the situation is worse because the phase velocity may, in some occasions, become negative. In that case, the wave blows up exponentially. Such waves are called 'backward' and render the PML ineffective. A spatial Fourier type analysis is now carried out for the convected wave equation in order to highlight the instability zones. 


\subsection{Plane wave stability analysis}

In free field, we assume the acoustic potential to be expressed as a plane wave of the form

$$
\varphi(x, y, \omega)=e^{-i k_{x} x-i k_{y} y} .
$$

Plugging this ansatz into the convected wave equation with zero right-hand side and $c_{0}$ to unity leads to

$$
\left(M_{x}^{2}-1\right) k_{x}^{2}+\left(M_{y}^{2}-1\right) k_{y}^{2}+2 M_{x} M_{y} k_{x} k_{y}-2 M_{x} k_{x} \omega-2 M_{y} k_{y} \omega+\omega^{2}=0,
$$

which is the Cartesian equation of an ellipse. The dispersion relation $D\left(\omega, k_{x}, k_{y}\right)=0$ sees the circular frequency $\omega(\boldsymbol{k})$ as a function of the propagating wavenumber $\boldsymbol{k}=\left(k_{x}, k_{y}\right)^{T}$, which we may write in terms of slowness vector

$$
D(1, S)=0, \quad S=\frac{k}{\omega}, \quad S=\left(\begin{array}{c}
S_{1} \\
S_{2}
\end{array}\right) .
$$

The set of points verifying the dispersion relation can be represented through the slowness diagram [22], which is shown in Figure 1.

(a) Dispersion relation $(2.13)$

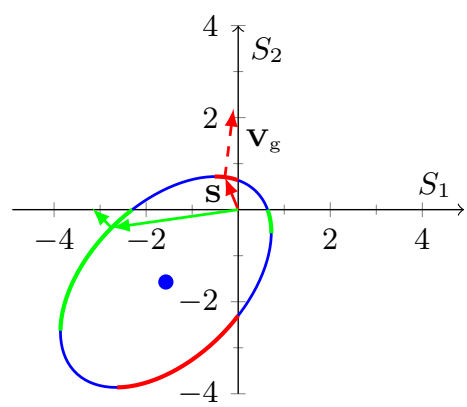

(b) After partial transform, eq (2.16)

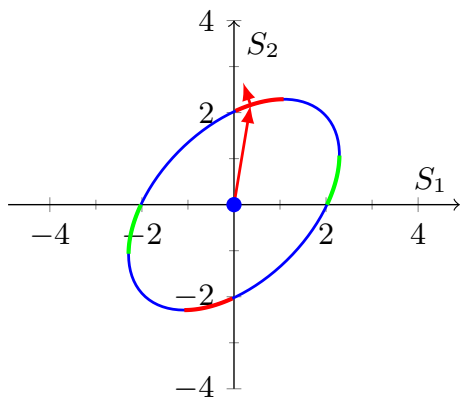

(c) After full transform, eq (2.17)

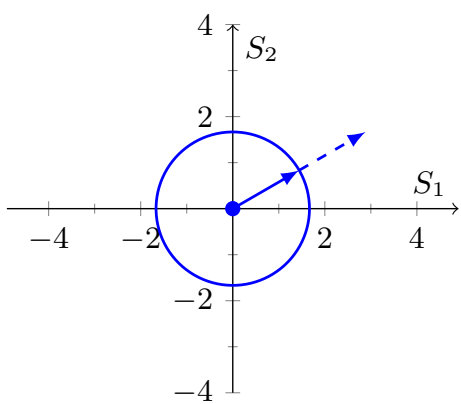

Figure 1: Slowness diagrams associated to different dispersion relations for a Mach number $M=0.8$ oriented at an angle $\theta=\pi / 4$. Stabilization process from left to right. The instability zones for a PML along the $x$-direction $(\longrightarrow)$ and $y$-direction $(-)$ are highlighted.

If the domain is bounded, the dispersion relation can exhibit multiple branches. This occurs for modal propagation, as we will see in Section 3.1. More complex behaviour, such as band gaps, are observed in metamaterials. At this point, it is useful to recall the definition of the phase and group velocity vectors $[22,29]$

$$
\boldsymbol{V}_{\mathrm{p}}(\omega(\boldsymbol{k}))=\frac{\omega(\boldsymbol{k})}{|\boldsymbol{k}|} \frac{\boldsymbol{k}}{|\boldsymbol{k}|}, \quad \boldsymbol{V}_{\mathrm{g}}(\boldsymbol{k})=\nabla_{\boldsymbol{k}} \omega(\boldsymbol{k})=-\left(\frac{\partial D}{\partial \omega}(\omega(\boldsymbol{k}), \boldsymbol{k})\right)^{-1} \nabla_{\boldsymbol{k}} D(\omega(\boldsymbol{k}), \boldsymbol{k}),
$$

so that the phase velocity points in the same direction as the slowness vector. In the slowness diagram, the group velocity $\boldsymbol{V}_{\mathrm{g}}$ is a vector pointing in the orthogonal direction to the slowness curve, and the slowness vector $\boldsymbol{S}$ points from the origin towards the slowness curve. The instability occurs when the slowness vector and group velocity are not oriented in the same way with respect to the PML direction. This is shown in Figure 1. More precisely, the stability condition for a PML in the $x$-direction is

$$
\forall \boldsymbol{k} \in \mathbb{R}^{2}, \quad\left(\boldsymbol{S} \cdot \boldsymbol{e}_{x}\right)\left(\boldsymbol{V}_{\mathrm{g}}(\omega(\boldsymbol{k})) \cdot \boldsymbol{e}_{x}\right) \geq 0,
$$

and similarly with $\boldsymbol{e}_{y}$ for a PML in the $y$-direction. We refer to [22, 38, 39] for additional details. In other words, the instability occurs when the phase and group velocities of the wave colliding the PML interface have opposite signs. We now apply the Lorentz transformation for the time variable only. In the Fourier space, it amounts to the substitution

$$
k_{x} \rightarrow k_{x}-\frac{\omega M_{x}}{\beta^{2}}, \quad k_{y} \rightarrow k_{y}-\frac{\omega M_{y}}{\beta^{2}},
$$


which removes the convective terms (related to the time-cross derivatives) in the convected Helmholtz equation. After the substitution, the dispersion relation reduces to

$$
\left(M_{x}^{2}-1\right) k_{x}^{2}+\left(M_{y}^{2}-1\right) k_{y}^{2}+2 M_{x} M_{y} k_{x} k_{y}+\frac{\omega^{2}}{\beta^{2}}=0 .
$$

The relation still describes an ellipse in the slowness diagram, but which is now centered at the origin (see Figure 1b). Applying the PML on the associated differential equation would still lead to instabilities, due to the presence of the mixed quadratic term. The second substitution acts on the spatial derivatives. In the Fourier space we have

$$
\begin{aligned}
& k_{x} \rightarrow\left(1+\frac{M_{x}^{2}}{\beta(1+\beta)}\right) k_{x}+\frac{M_{x} M_{y}}{\beta(1+\beta)} k_{y}, \\
& k_{y} \rightarrow \frac{M_{x} M_{y}}{\beta(1+\beta)} k_{x}+\left(1+\frac{M_{y}^{2}}{\beta(1+\beta)}\right) k_{y},
\end{aligned}
$$

which in the slowness diagram, rotates and shrinks the ellipse onto a circle of radius $1 / \beta$, see Figure $1 \mathrm{c}$. The new dispersion relation

$$
-k_{x}^{2}-k_{y}^{2}+\frac{\omega^{2}}{\beta^{2}}=0
$$

describes an isotropic medium. By reducing the convected Helmholtz equation into a classical Helmholtz equation, the Lorentz transformation has removed all possible instabilities.

\subsection{Summing up}

The classical PML can be unstable due to the anisotropic nature of convected propagation. It occurs when the phase and group velocities of the wave hitting the PML are of opposite signs. As will be seen further, such instabilities can be of moderate growth or even insignificant for some specific PML configurations. However, the technique is not reliable in the general case. Through the Lorentz transformation, the convected Helmholtz equation is reduced to a classical Helmholtz equation with an effective wavenumber. The idea is then to apply the usual PML on this modified equation, as one would do for Helmholtz problems. Finally, the physical variables are retrieved by performing the inverse Lorentz transformation. This leads to a new PML model, which coincides with the convected Helmholtz equation in the physical domain. The global procedure is summarized in Figure 2. The derivation of the Lorentz transformed PML is now presented on several practical examples of increasing complexity.

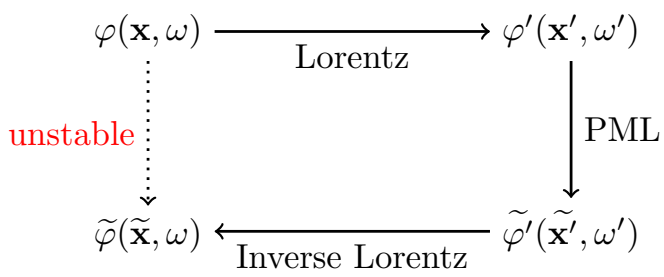

Figure 2: Summary of the procedure to derive a stable PML formulation.

\section{Stabilized formulation for a uniaxial flow}

Let us consider a quasi one-dimensional situation where the mean flow and PML are uniaxial $\left(M_{y}=0\right)$. This model is suitable to describe convected modal propagation in a straight duct. The Lorentz transformation in the frequency domain takes the simple form

$$
\varphi(x, y, \omega)=\frac{1}{\beta} \varphi^{\prime}\left(x^{\prime}, y^{\prime}, \omega^{\prime}\right) e^{i k_{0}^{\prime} M x^{\prime}}, \quad\left(x^{\prime}, y^{\prime}\right)=\left(\frac{x}{\beta}, y\right), \quad k_{0}^{\prime}=\frac{k_{0}}{\beta},
$$


and the partial derivatives are

$$
\frac{\partial}{\partial x}=\frac{1}{\beta}\left(\frac{\partial}{\partial x^{\prime}}+i k_{0}^{\prime} M\right), \quad \frac{\partial}{\partial y}=\frac{\partial}{\partial y^{\prime}} .
$$

The convected Helmholtz equation becomes a Helmholtz equation in the Lorentz space, where we can apply the complex stretching as defined in Section 2.2

$$
\left\{\frac{\partial^{2}}{\partial \widetilde{x^{\prime}}}+\frac{\partial^{2}}{\partial \widetilde{y^{\prime}}}+k_{0}^{\prime 2}\right\} \widetilde{\varphi^{\prime}}\left(\widetilde{x^{\prime}}, \widetilde{y^{\prime}}, \omega^{\prime}\right)=-f^{\prime}\left(x^{\prime}, y^{\prime}, \omega^{\prime}\right) \text { in } \Omega^{\prime}
$$

where $f^{\prime}$ is the distribution of volume sources in the Lorentz space. We use the notations $\Omega^{\prime}$ and $\Omega_{\text {phy }}^{\prime}$ to refer respectively to the global and physical domains in the Lorentz space. Since we consider waves traveling along the $x$-direction only, we set $\gamma_{y}=1$ and develop the differential operator as

$$
\left\{\gamma_{x}^{-1} \frac{\partial}{\partial x^{\prime}}\left(\gamma_{x}^{-1} \frac{\partial}{\partial x^{\prime}}\right)+\frac{\partial^{2}}{\partial y^{\prime 2}}+k_{0}^{\prime 2}\right\} \tilde{\varphi}^{\prime}=-f^{\prime} \quad \text { in } \Omega^{\prime}
$$

We now need to revert to the physical variables $(x, y, \omega)$ by using the inverse Lorentz transformation. Doing so implicitly ensures the continuity of the formulation between $\Omega_{\mathrm{phy}}$ and $\Omega_{\mathrm{PML}}$. Inverting the operators in (3.1) gives

$$
\gamma_{x}^{-1} \frac{\partial}{\partial x^{\prime}}=\gamma_{x}^{-1} \beta\left(\frac{\partial}{\partial x}-\frac{i k_{0} M}{\beta^{2}}\right), \quad \frac{\partial}{\partial y^{\prime}}=\frac{\partial}{\partial y},
$$

and the second order $x^{\prime}$-derivative is developed as

$$
\begin{aligned}
\frac{\partial}{\partial x^{\prime}}\left(\gamma_{x}^{-1} \frac{\partial}{\partial x^{\prime}}\right) & =\beta \frac{\partial}{\partial x^{\prime}}\left(\gamma_{x}^{-1}\left(\frac{\partial}{\partial x}-\frac{i k_{0} M}{\beta^{2}}\right)\right) \\
& =\beta^{2}\left(\frac{\partial}{\partial x}\left(\gamma_{x}^{-1} \frac{\partial}{\partial x}-\gamma_{x}^{-1} \frac{i k_{0} M}{\beta^{2}}\right)-\frac{i k_{0} M}{\beta^{2}}\left(\gamma_{x}^{-1} \frac{\partial}{\partial x}-\gamma_{x}^{-1} \frac{i k_{0} M}{\beta^{2}}\right)\right) \\
& =\beta^{2} \frac{\partial}{\partial x}\left(\gamma_{x}^{-1} \frac{\partial}{\partial x}\right)-2 \gamma_{x}^{-1} i k_{0} M \frac{\partial}{\partial x}-\gamma_{x}^{-1} \frac{k_{0}^{2} M^{2}}{\beta^{2}}-i k_{0} M\left(\partial_{x} \gamma_{x}^{-1}\right) .
\end{aligned}
$$

After multiplication by $e^{i k_{0}^{\prime} M x^{\prime}}$ and $\gamma_{x}$, equation (3.3) leads to the stabilized PML model in the physical variables

$$
\left(1-M^{2}\right) \frac{\partial}{\partial x}\left(\gamma_{x}^{-1} \frac{\partial \widetilde{\varphi}}{\partial x}\right)-2 i k_{0} M \gamma_{x}^{-1} \frac{\partial \widetilde{\varphi}}{\partial x}+\left(\frac{k_{0}^{2}\left(\gamma_{x}-\gamma_{x}^{-1} M^{2}\right)}{\left(1-M^{2}\right)}-i k_{0} M\left(\partial_{x} \gamma_{x}^{-1}\right)\right) \widetilde{\varphi}+\gamma_{x} \frac{\partial^{2} \widetilde{\varphi}}{\partial y^{2}}=-f \quad \text { in } \Omega
$$

When $\gamma_{x}=1$, we immediately recover the usual convected equation

$$
\left(1-M^{2}\right) \partial_{x}^{2} \varphi+\partial_{y}^{2} \varphi-2 i k_{0} M \partial_{x} \varphi+k_{0}^{2} \varphi=-f \quad \text { in } \Omega_{\text {phy }} .
$$

\subsection{Modal propagation in a straight two-dimensional duct}

A boundary value problem associated to the model (3.5) is formulated. The setup and boundary conditions are defined in a truncated, connected semi-infinite duct specified in Figure 3. An acoustic duct mode is injected on $\Gamma_{1}$ along the $x$-direction and homogeneous Neumann boundary conditions are used on the upper and lower boundaries denoted $\Gamma_{2}$. A PML is appended at the outlet, at the end of which a homogeneous Neumann boundary condition is prescribed.

In the physical domain, the acoustic field is the homogeneous solution of (3.6), and can be formulated into right-propagating duct modes as follows

$$
\varphi_{\mathrm{ex}}(x, y)=A \cos \left(k_{y} y\right) e^{-i k_{x} x}, \quad k_{y}=\frac{n \pi}{H}, \quad A \in \mathbb{C}, \quad n \in \mathbb{N}, \quad(x, y) \in \Omega_{\mathrm{phy}},
$$




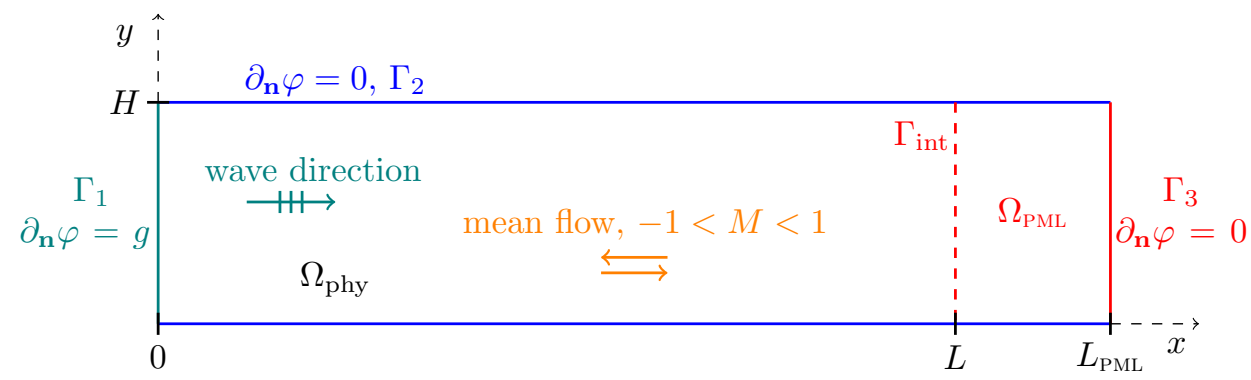

Figure 3: Sketch of the numerical case: $2 \mathrm{D}$ acoustic duct propagation in a uniform mean flow. The PML interface is $\Gamma_{\text {int }}=$ $\overline{\Omega_{\mathrm{phy}}} \cap \overline{\Omega_{\mathrm{PML}}}$ and the global domain $\Omega$ is the open set such that $\bar{\Omega}=\overline{\Omega_{\mathrm{phy}}} \cup \overline{\Omega_{\mathrm{PML}}}$. The PML outer boundary is $\Gamma_{3}$ and $\mathbf{n}$ is the outward normal.

where the wavenumber $k_{x}$ is given by the dispersion relation

$$
\begin{cases}k_{x}=\frac{1}{1-M^{2}}\left(-M k_{0}+\sqrt{k_{0}^{2}-\left(1-M^{2}\right) k_{y}^{2}}\right), & \text { if } k_{0}^{2}>\left(1-M^{2}\right) k_{y}^{2}, \\ k_{x}=\frac{1}{1-M^{2}}\left(-M k_{0}-i \sqrt{\left(1-M^{2}\right) k_{y}^{2}-k_{0}^{2}}\right), & \text { if } k_{0}^{2}<\left(1-M^{2}\right) k_{y}^{2},\end{cases}
$$

depending on the sign of the square-root term, which is here chosen such that the modes are outgoing. The general solution of the duct problem involves a linear superposition of all modes, see e.g [40]. The values taken by $k_{x}$ describe respectively propagative (cut-on) and evanescent (cut-off) modes. Since the domain is bounded in the $y$-direction, the $k_{y}$ values are discrete. The plane wave $(n=0)$ is always propagative and the new branches of the dispersion relations, called modes, propagate when $k_{0}>\sqrt{1-M^{2}} k_{y}$.

We can use the analysis carried out in Section 2.3 to understand the PML instability issue. Here, the propagation only occurs along the positive half-plane. For propagating waves, the phase velocity is the inverse of the slowness vector, which is

$$
V_{\mathrm{p}}=\frac{k_{0}}{k_{x}}=\frac{\left(1-M^{2}\right) k_{0}}{-M k_{0}+\sqrt{k_{0}^{2}-\left(1-M^{2}\right)\left(\frac{n \pi}{H}\right)^{2}}},
$$

and the group velocity is

$$
V_{\mathrm{g}}=\frac{\partial k_{0}}{\partial k_{x}}=\frac{\left(1-M^{2}\right) \sqrt{k_{0}^{2}-\left(1-M^{2}\right)\left(\frac{n \pi}{H}\right)^{2}}}{k_{0}-M \sqrt{k_{0}^{2}-\left(1-M^{2}\right)\left(\frac{n \pi}{H}\right)^{2}}} .
$$

When $M>0$ and $n \neq 0$, the wave may have a positive group velocity but negative phase velocity. This happens if $k_{0}$ lies in the range

$$
\sqrt{1-M^{2}}\left(\frac{n \pi}{H}\right)<k_{0}<\frac{n \pi}{H}
$$

We then distinguish two types of propagating regimes by increasing values of $k_{0}$. They will be respectively referred to as inverse upstream and propagative. The group and phase velocities for three distinct modes are represented in Figure 4a, where both regimes are emphasized. Note that there is no instability when $M<0$. We do not discuss the behaviour on evanescent modes since the PML only adds an oscillatory part to the solution [9], which remains true for the convected problem.

For high frequencies, i.e. high values of $k_{0}$, the phase and group velocities tend to be those of the plane wave

$$
V_{\mathrm{g}}=V_{\mathrm{p}}=(1+M) .
$$

If we now consider the stabilized PML model, it can be shown that

$$
\widetilde{\varphi}(x, y)=A \cos \left(k_{y} y\right) e^{-i k_{x} x} e^{-\frac{k_{x}^{\prime}}{\beta k_{0}} \zeta(x)}=A \cos \left(k_{y} y\right) e^{-i k_{x} x} e^{-\frac{\tilde{k}_{x}}{k_{0}} \zeta(x)}, \quad(x, y) \in \Omega,
$$


(a) Physical wave properties

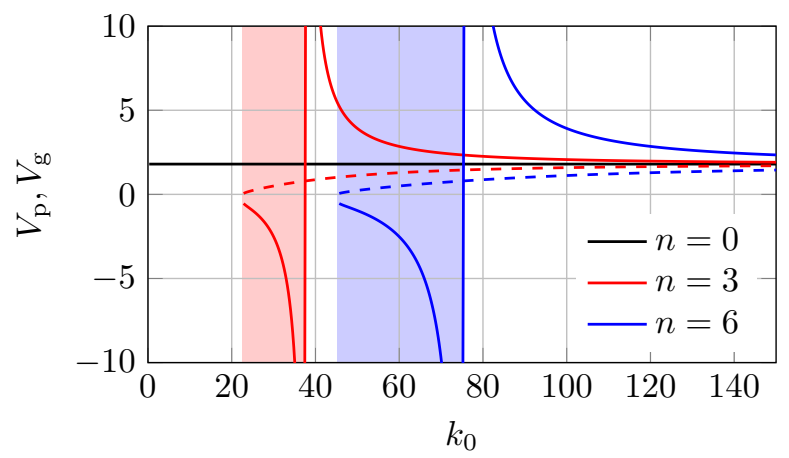

(b) effective PML wave properties

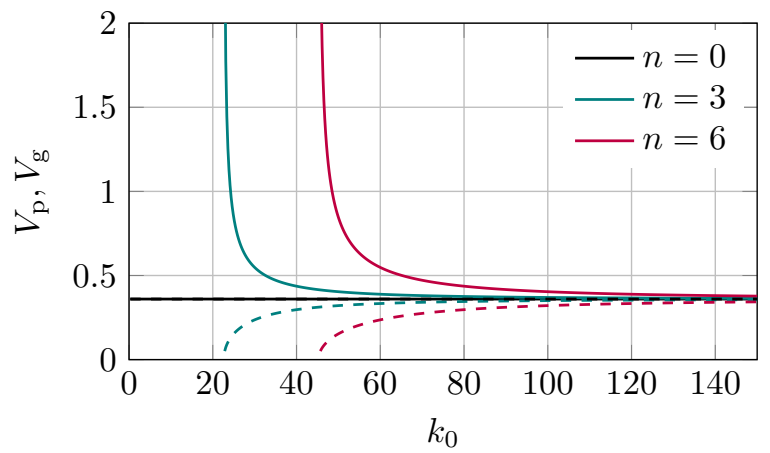

Figure 4: Phase $V_{\mathrm{p}}$ (plain lines) and group $V_{\mathrm{g}}$ (dashed lines) velocities for three propagative modes $n=\{0,3,6\}$ and $M=0.8$. The shaded areas highlight the inverse upstream regime.

satisfies equation (3.5). The modified wavenumber $\widetilde{k}_{x}$ that is seen by the PML

$$
\widetilde{k}_{x}=\frac{k_{x}^{\prime}}{\beta}=\frac{1}{\beta} \sqrt{{k_{0}^{\prime}}^{2}-k_{y}^{2}}=\frac{\sqrt{k_{0}^{2}-\left(1-M^{2}\right) k_{y}^{2}}}{1-M^{2}},
$$

is always positive for propagative modes. The convective instability, coming from the shift by $-M k_{0} / \beta^{2}$ in relation (3.8), has been removed from the PML point of view, see Figure 4b. We now present how to set up the weak formulation of this model for a use in a finite element context.

\subsection{Weak formulation}

In order to build the weak formulation, we start from the transformed Helmholtz-PML equation (3.3). For conciseness, we omit the $\widetilde{r}$ notation when writing weak formulations. We set $f^{\prime}=0$ since the source is coming from the boundary condition on $\Gamma_{1}$. After multiplying the equation by $\gamma_{x}$ and using Green's formula, one obtains for $\varphi^{\prime} \in H^{1}\left(\Omega^{\prime}\right)$

$$
\begin{array}{r}
\forall \psi^{\prime} \in H^{1}\left(\Omega^{\prime}\right), \quad \int_{\Omega^{\prime}}\left(\gamma_{x}^{-1} \partial_{x^{\prime}} \varphi^{\prime} \overline{\partial_{x^{\prime}} \psi^{\prime}}+\gamma_{x} \partial_{y^{\prime}} \varphi^{\prime} \overline{\partial_{y^{\prime}} \psi^{\prime}}-\gamma_{x} k_{0}^{\prime 2} \varphi^{\prime} \overline{\psi^{\prime}}\right) d \Omega^{\prime} \\
=\int_{\partial \Omega^{\prime}}\left(\gamma_{x}^{-1} \partial_{n_{x}^{\prime}} \varphi^{\prime} \overline{\psi^{\prime}}+\gamma_{x} \partial_{n_{y}^{\prime}} \varphi^{\prime} \overline{\psi^{\prime}}\right) d s^{\prime},
\end{array}
$$

where $n_{x}^{\prime}$ and $n_{y}^{\prime}$ are the normal unit vectors with respect to the $x^{\prime}$ - and $y^{\prime}$-axes. The boundary integral may be split as

$$
\int_{\partial \Omega^{\prime}}\left(\gamma_{x}^{-1} \partial_{n_{x}^{\prime}} \varphi^{\prime} \overline{\psi^{\prime}}+\gamma_{x} \partial_{n_{y}^{\prime}} \varphi^{\prime} \overline{\psi^{\prime}}\right) d s^{\prime}=\int_{\Gamma_{1} \cup \Gamma_{3}} \gamma_{x}^{-1} \partial_{n_{x}^{\prime}} \varphi^{\prime} \overline{\psi^{\prime}} d y^{\prime}+\int_{\Gamma_{2}} \gamma_{x} \partial_{n_{y}^{\prime}} \varphi^{\prime} \overline{\psi^{\prime}} d x^{\prime} .
$$

Regarding the volume integral, the $x^{\prime}$-derivative product of the trial and test functions becomes

$$
\begin{aligned}
\partial_{x^{\prime}} \varphi^{\prime} \overline{\partial_{x^{\prime}} \psi^{\prime}} & =\left(\beta \partial_{x} \varphi-\frac{i k_{0} M}{\beta} \varphi\right) e^{i k_{0}^{\prime} M x^{\prime}}\left(\beta \overline{\partial_{x} \psi}+\frac{i k_{0} M}{\beta} \bar{\psi}\right) e^{-i k_{0}^{\prime} M x^{\prime}} \\
& =\beta^{2} \partial_{x} \varphi \overline{\partial_{x} \psi}-i k_{0} M \varphi \overline{\partial_{x} \psi}+i k_{0} M \partial_{x} \varphi \bar{\psi}+\frac{k_{0}^{2} M^{2}}{\beta^{2}} \varphi \bar{\psi}
\end{aligned}
$$

The coordinates in the Lorentz space are stretched by the Jacobian matrix of the transformation from $\Omega^{\prime}$ to $\Omega$ as

$$
\boldsymbol{L}=\left(\begin{array}{ll}
\frac{\partial x^{\prime}}{\partial x} & \frac{\partial x^{\prime}}{\partial y} \\
\frac{\partial y^{\prime}}{\partial x} & \frac{\partial y^{\prime}}{\partial y}
\end{array}\right)=\left(\begin{array}{cc}
1 / \beta & 0 \\
0 & 1
\end{array}\right)
$$


so that $d \Omega^{\prime}=\operatorname{det}(\boldsymbol{L}) d \Omega=\frac{1}{\beta} d \Omega$. The formulation (3.13) can be rewritten in the physical variables, for $\varphi \in H^{1}(\Omega)$, as

$$
\begin{aligned}
\forall \psi \in H^{1}(\Omega), & \int_{\Omega} \gamma_{x}^{-1}\left(\beta^{2} \partial_{x} \varphi \overline{\partial_{x} \psi}-i k_{0} M \varphi \overline{\partial_{x} \psi}+i k_{0} M \partial_{x} \varphi \bar{\psi}+\frac{k_{0}^{2} M^{2}}{\beta^{2}} \varphi \bar{\psi}\right) \frac{1}{\beta} d \Omega \\
+ & \int_{\Omega} \gamma_{x}\left(\partial_{y} \varphi \overline{\partial_{y} \psi}-\frac{k_{0}^{2}}{\beta^{2}} \varphi \bar{\psi}\right) \frac{1}{\beta} d \Omega=\int_{\Gamma_{1} \cup \Gamma_{3}} \gamma_{x}^{-1}\left(\beta \partial_{n_{x}} \varphi-\frac{i k_{0} M_{n_{x}}}{\beta} \varphi\right) \bar{\psi} d y+\int_{\Gamma_{2}} \gamma_{x} \partial_{n_{y}} \varphi \bar{\psi} \frac{1}{\beta} d x,
\end{aligned}
$$

where $M_{n_{x}}= \pm M$ depending on the orientation of the normal $n_{x}$. The boundary integrals on $\Gamma_{3}$ and along the $y$-direction vanish because homogeneous Neumann boundary conditions are imposed on $x=L_{\mathrm{PML}}, y=0$ and $y=H$. After multiplication by $\beta$, we obtain on the input boundary $\Gamma_{1}$ the same boundary integral that one would derive for the usual convected Helmholtz problem without PML

$$
\int_{\Gamma_{1}}\left(\beta^{2} \partial_{n_{x}} \varphi \bar{\psi}-i k_{0} M_{n_{x}} \varphi \bar{\psi}\right) d y=\int_{\Gamma_{1}}\left(\beta^{2} g \bar{\psi}+i k_{0} M \varphi \bar{\psi}\right) d y
$$

Finally, the stable weak formulation for the boundary value problem from Figure 3 states

$$
\left\{\begin{array}{l}
\text { Find } \varphi \in H^{1}(\Omega) \text { such that, } \forall \psi \in H^{1}(\Omega), \\
\beta^{2} \int_{\Omega} \gamma_{x}^{-1} \partial_{x} \varphi \overline{\partial_{x} \psi} d \Omega+i k_{0} M \int_{\Omega} \gamma_{x}^{-1}\left(\partial_{x} \varphi \bar{\psi}-\varphi \overline{\partial_{x} \psi}\right) d \Omega-\frac{k_{0}^{2}}{\beta^{2}} \int_{\Omega}\left(\gamma_{x}-\gamma_{x}^{-1} M^{2}\right) \varphi \bar{\psi} d \Omega \\
+\int_{\Omega} \gamma_{x} \partial_{y} \varphi \overline{\partial_{y} \psi} d \Omega=i k_{0} M \int_{\Gamma_{1}} \varphi \bar{\psi} d y+\beta^{2} \int_{\Gamma_{1}} g \bar{\psi} d y .
\end{array}\right.
$$

When $\gamma_{x}=1$, we retrieve the volume terms from the usual convected Helmholtz problem. On the interface between the physical and PML domain we have the continuity requirements

$$
\varphi, \gamma_{x}^{-1} \partial_{n_{x}} \varphi \text { continuous at } x=L .
$$

The continuity of $\varphi$ in $\Omega$ is ensured by construction of the weak formulation. The same can be said for the normal derivative if we set $\gamma_{x}=1$ at $x=L$. With finite elements, the continuity of $\varphi$ is implicitly enforced by considering one coincident nodal value (i.e. by merging nodes) at the interface vertices. In this PML model, the integrals involved for the construction of the mass matrix are coupled with the function $\gamma_{x}$. Note that an additional application of Green's formula allows to exactly recover the terms of equation (3.5).

\subsection{Alternative formulation}

The model presented above is not the only way to obtain a stable PML. Another possibility, described by Bécache et al. [16], consists in transforming the convected Helmholtz equation in the PML domain only. The procedure is shown in Figure 5. The method actually corresponds to a Lorentz transformation except that there is no contraction factor in the time-domain

$$
t^{*}=t+\frac{M}{\beta^{2} c_{0}} x .
$$

In the frequency domain ( $e^{i \omega t}$ convention), one goes from the physical space to the transformed space thanks to the substitution of the spatial partial derivative

$$
\partial_{x} \rightarrow \partial_{x^{*}}+i \lambda(x), \quad \lambda(x)=\left\{\begin{array}{l}
\frac{k_{0} M}{1-M^{2}} \text { in } \Omega_{\mathrm{PML}}, \\
0 \quad \text { otherwise }
\end{array}\right.
$$

This alternative transformation is based on the analysis of the dispersion relation (3.8) to remove the presence of unstable modes. It gives two different weak statements:

- in the physical domain, the weak formulation associated to the usual convected Helmholtz problem

$$
\begin{aligned}
\forall \psi \in H^{1}\left(\Omega_{\mathrm{phy}}\right),\left(1-M^{2}\right) & \int_{\Omega_{\mathrm{phy}}} \partial_{x} \varphi \overline{\partial_{x} \psi}+\int_{\Omega_{\mathrm{phy}}} \partial_{y} \varphi \overline{\partial_{y} \psi}+i k_{0} M \int_{\Omega_{\mathrm{phy}}}\left(\partial_{x} \varphi \bar{\psi}-\varphi \overline{\partial_{x} \psi}\right) \\
& -k_{0}^{2} \int_{\Omega_{\mathrm{phy}}} \varphi \bar{\psi}=i k_{0} M \int_{\Gamma_{1}} \varphi \bar{\psi}+\beta^{2} \int_{\Gamma_{1}} g \bar{\psi}
\end{aligned}
$$


- and in the PML domain, plugging (3.16) into (3.17) gives the problem in the transformed space

$$
\begin{aligned}
& \forall \psi^{*} \in H^{1}\left(\Omega_{\mathrm{PML}}\right),\left(1-M^{2}\right) \int_{\Omega_{\mathrm{PML}}} \gamma_{x}^{-1} \partial_{x^{*}} \varphi^{*} \overline{\partial_{x^{*}} \psi^{*}}+\int_{\Omega_{\mathrm{PML}}} \gamma_{x} \partial_{y^{*}} \varphi^{*} \overline{\partial_{y^{*}} \psi^{*}} \\
& -\frac{k_{0}^{2}}{1-M^{2}} \int_{\Omega_{\mathrm{PML}}} \gamma_{x} \varphi^{*} \overline{\psi^{*}}=0,
\end{aligned}
$$

where we have denoted by $\varphi^{*}$ the alternative PML solution. Note that this transformation does not 'stretch' the space and we write directly $\Omega$ instead of $\Omega^{*}$. We may associate to equation (3.18) the strong form

$$
\left(1-M^{2}\right) \partial_{x^{*}}^{2} \widetilde{\varphi^{*}}+\partial_{y^{*}}^{2} \widetilde{\varphi^{*}}+\frac{k_{0}^{2}}{1-M^{2}} \widetilde{\varphi^{*}}=0, \quad \text { in } \Omega_{\mathrm{PML}} .
$$

In comparison to the exact solution obtained through the Lorentz transform in (3.11), we can see that

$$
\widetilde{\varphi}=e^{i \frac{k_{0} M}{1-M^{2}} x} \widetilde{\varphi^{*}} \text { in } \Omega_{\mathrm{PML}},
$$

and remark that both solutions differ by a phase factor in $\Omega_{\mathrm{PML}}$. This is summarized in Table 1 , which provides the continuous (exact) solutions of the plane wave mode in $\Omega_{\text {phy }}$ and $\Omega_{\mathrm{PML}}$ for each PML formulation. It turns out that the Lorentz and alternative formulations coincide if one imposes the jump condition defined in [16, eq. (3.13)] on the PML interface. However, the jump condition acts on the normal derivative and as a result, cannot be enforced in a conventional $H^{1}$-conformal finite element basis. Although such a condition can be used in the time domain [21], we will see that it is not a strict requirement in the frequency domain.

$$
\varphi(\mathbf{x}, \omega) \underset{\text { Transformation }}{\longrightarrow} \varphi^{*}\left(\mathbf{x}^{*}, \omega\right) \underset{\text { PML }}{\longrightarrow} \widetilde{\varphi^{*}}\left(\widetilde{\mathbf{x}^{*}}, \omega\right)
$$

Figure 5: Summary of the alternative stable procedure in $\Omega_{\mathrm{PML}}$. Note that the two steps of the procedure are independent.

Table 1: Summary of the $x$-dependence of the exact solutions $\widetilde{\varphi}$ for the three PML formulations.

\begin{tabular}{ccc} 
Model & $\Omega_{\mathrm{phy}}$ & $\Omega_{\mathrm{PML}}$ \\
\hline Classical & $e^{-i k_{x} x}$ & $e^{-i k_{x} x} e^{-\frac{k_{x}}{k_{0}} \zeta(x)}$ \\
Alternative & $e^{-i k_{x} x}$ & $e^{-i \widetilde{k}_{x} x} e^{-\frac{\tilde{k}_{x}}{k_{0}} \zeta(x)}$ \\
Lorentz & $e^{-i k_{x} x}$ & $e^{-i k_{x} x} e^{-\frac{\tilde{k}_{x}}{k_{0}} \zeta(x)}$
\end{tabular}

\subsection{Numerical illustrations}

In this section, we present the numerical properties of the introduced models for a broadband frequency range, and illustrate their convergence properties. The numerical results have been obtained with the mesh generator Gmsh [41] and an in-house Matlab finite element implementation. We use a high-order finite element scheme equipped with a basis of integrated Legendre polynomials [42] to discretize the weak formulations. The $p$-FEM is less sensitive to dispersion errors and has shown to provide substantial reductions in memory and CPU time when compared to conventional low-order FEM on both Helmholtz [43] and convected Helmholtz [25] applications. The integration on the reference element is computed by a tensorised Gauss quadrature rule with $(p+1)^{2}$ points, where $p$ is the order of the integrated Legendre polynomial shape functions.

As a reference solution, the solution obtained from the exact non-reflecting boundary condition at the duct output boundary is used. This reference solution gives a useful insight into the different error contributions, as it allows to separate the PML induced errors from the $p$-FEM discretization errors. It is given by the Dirichlet-to-Neumann (DtN) operator, which may write for a single mode

$$
\partial_{x} \varphi_{\mathrm{ref}}=-i k_{x} \varphi_{\mathrm{ref}}, \quad \text { at } x=L .
$$


It follows the discretization of a one-dimensional mass matrix. The relative $L^{2}$-error is recorded in $\Omega_{\text {phy }}$ as

$$
\mathcal{E}_{L^{2}}=100 \frac{\left\|\varphi_{\mathrm{ex}}-\varphi_{h}\right\|_{L^{2}\left(\Omega_{\mathrm{phy}}\right)}}{\left\|\varphi_{\mathrm{ex}}\right\|_{L^{2}\left(\Omega_{\mathrm{phy}}\right)}}
$$

where $\varphi_{h}$ refers to the discretized solution and $\varphi_{\text {ex }}$ to the exact solution (3.7). For convected applications, the resulting global error shares the same features as Helmholtz problems [44], and exponential convergence is expected under $p$-refinements for smooth solutions. The rectangular duct is chosen to be of size $L=0.5$ and $H=0.25$. Linear quadrangle elements Q4 are used to mesh the physical domain $\Omega_{\text {phy }}$. The domain $\Omega_{\mathrm{PML}}$ is automatically extruded with 4 layers of Q4 elements. The width of each layer is equal to the mesh size of the physical domain, chosen for instance as

$$
h=\frac{1}{20} \sqrt{L H} .
$$

We use the unbounded function (2.9) as absorbing function. The parameter $\sigma_{0}$ could be selected, for instance, to match the phase velocity seen by the PML in the high frequency approximation

$$
\begin{aligned}
\sigma_{0} & =\frac{k_{0}}{k_{x}} \underset{k_{0} \rightarrow+\infty}{=}(1+M) \quad \text { classical PML, } \\
\sigma_{0} & =\frac{k_{0}}{\widetilde{k}_{x}} \underset{k_{0} \rightarrow+\infty}{=}\left(1-M^{2}\right)=\beta^{2} \quad \text { Lorentz and alternative PMLs, }
\end{aligned}
$$

in such a way that the decay of plane waves in the PML is linear, resulting in a reflectionless layer. In practice, it is not clear how to choose $\sigma_{0}$ because the phase velocity seen by the PML depends directly on the input mode, the mean flow, the input frequency, and becomes large close to cut-off (see Figure 4). More precisely, one can notice from equation (2.11) that the amplitude of $\widetilde{\varphi}$ behaves in the layer as

$$
(1-x)^{\alpha}, x \in[0,1], \quad \begin{cases}\alpha>1, & \text { if } \sigma_{0}>V_{\mathrm{p}}, \\ \alpha<1, & \text { if } \sigma_{0}<V_{\mathrm{p}}, \\ \alpha=1, & \text { if } \sigma_{0}=V_{\mathrm{p}},\end{cases}
$$

where we emphasize that $V_{\mathrm{p}}$ differs whether the classical or stable PML is used. As a result, our method is expected to converge exponentially under $p$-refinement only if $\sigma_{0}>V_{\mathrm{p}}$, and will be algebraic otherwise [45], driven by the ratio between $\sigma_{0}$ and $V_{\mathrm{p}}$. In the experiments, we fix $\sigma_{0}=4 \beta^{2}$ to ensure a good convergence rate for all models in the selected frequency range. Appendix A examines more precisely the role of $\sigma_{0}$ on the convergence rate of the PML. Here, we do not aim to optimize the discrete properties, but rather focus on the formulations. More details on the optimization of the PML in discrete contexts can be found in [5, 6]. Note that a precise tuning of the PML mesh can largely improve its efficiency. Some guidelines can be found for instance in $[46,47,48]$ and a promising approach based on $h p$-adaptivity has been studied in [49].

Let us consider two frequencies $k_{0}=30$ and $k_{0}=70$, respectively in the inverse upstream and propagative regimes, for the third mode $n=3$ and a mean flow $M=0.8$. The propagation occurs along the positive $x$-direction. Figures 6 and 7 illustrate the differences between the three PML models. When the wave is inverse upstream, the classical PML shows an exponential growth in the layer. Both stable formulations correct this unsought growth by making the mode evanescent in the PML, and the $L^{2}$-error is close to the one from the reference solution. When the wave is propagative, the alternative and Lorentz models show a different absorption behaviour in $\Omega_{\mathrm{PML}}$. As seen by the analysis at the continuous level, there is a phase shift mismatch between $\Omega_{\text {phy }}$ and $\Omega_{\mathrm{PML}}$ for the alternative formulation: the frequency is higher in $\Omega_{\mathrm{PML}}$ than in $\Omega_{\text {phy }}$, thus leading to a higher discretization error and numerical reflections. This confirms the results from Table 1.

We further report the $L^{2}$-error for a broadband frequency range in Figure 8. The results are shown for the third and sixth modes. For both modes, the Lorentz model is close to the reference solution. The classical PML suffers from instability in the inverse upstream regime and the alternative PML from discretization errors since the frequency is higher in $\Omega_{\mathrm{PML}}$. This phenomenon increases for high-order modes, as the number of oscillations grows in the $y$-direction. 
$\mathcal{E}_{L^{2}}=1.0 \mathrm{e}+2 \%$

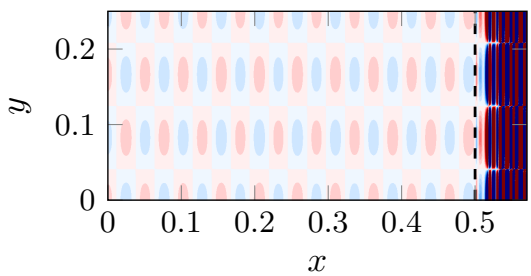

(a) Classical
$\mathcal{E}_{L^{2}}=1.1 \mathrm{e}-4 \%$

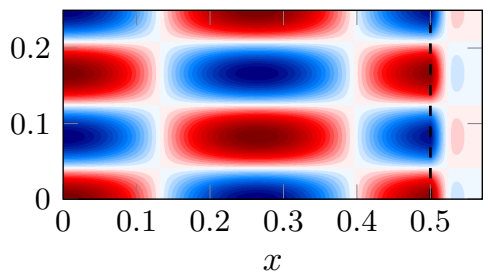

(b) Alternative

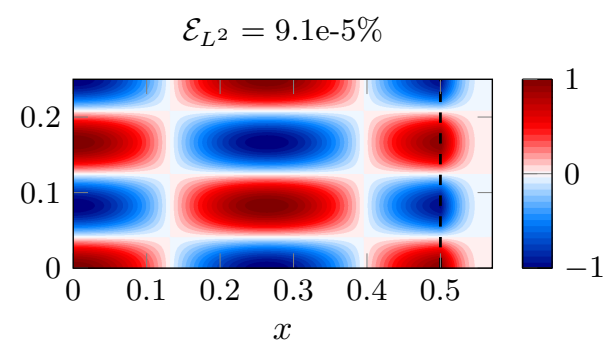

(c) Lorentz

Figure 6: Inverse upstream mode: $k_{0}=30$. Real part of the numerical solution for the three formulations. Reference $L^{2}$-error: $9.1 \times 10^{-5} \%$. Mach number $M=0.8$, input mode $n=3$, shape function order $p=4$. The dashed line $(---)$ is the PML interface.

$\mathcal{E}_{L^{2}}=7.4 \mathrm{e}-3 \%$

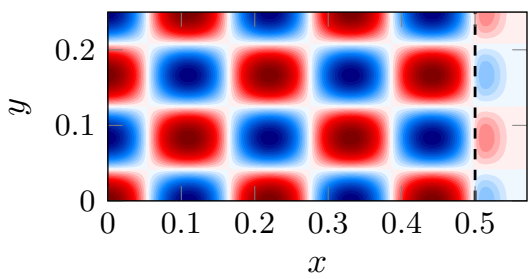

(a) Classical
$\mathcal{E}_{L^{2}}=6.0 \mathrm{e}-1 \%$

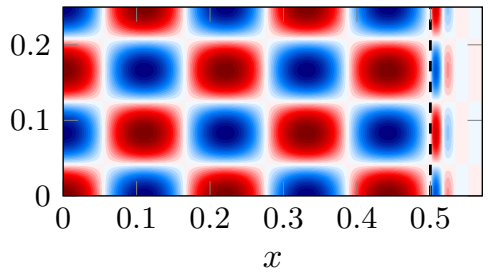

(b) Alternative
$\mathcal{E}_{L^{2}}=1.5 \mathrm{e}-2 \%$

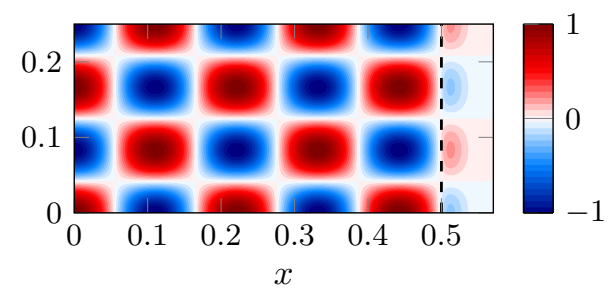

(c) Lorentz

Figure 7: Propagative mode: $k_{0}=70$. Real part of the numerical solution for the three formulations. Reference $L^{2}$-error: $1.3 \times 10^{-3} \%$. Mach number $M=0.8$, input mode $n=3$, shape function order $p=4$. The dashed line $(---)$ is the PML interface.
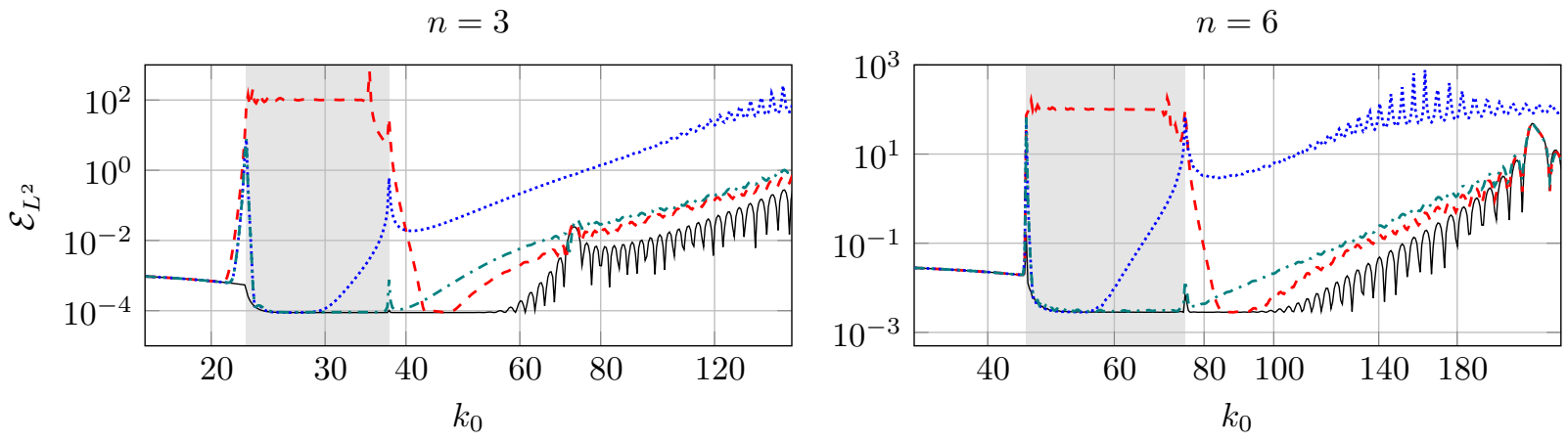

Figure 8: $L^{2}$-error (in \%) for the classical (- - ) , alternative (.........) and Lorentz (-.-.-) PMLs. Parameters: $M=0.8, p=4$ and $\sigma_{0}=4 \beta^{2}$. The shaded areas highlight inverse upstream propagation. Reference solution $(-)$.

As mentioned above, exponential $p$-convergence is expected for smooth solutions. Figure 9 reports the influence of the shape function polynomial order $p$ on the $L^{2}$-error. When the wave is inverse upstream, at $k_{0}=30$, the usual PML is unstable and the convergence is hampered. Both stable formulations fit the error from the exact non-reflecting condition when $p$ increases, thus confirming their effectiveness in this regime. At $k_{0}=70$, the errors from the classical and Lorentz methods are close to the one from the reference solution. The alternative formulation has a comparable convergence rate, although the discretization error is higher. An optimal convergence rate is observed for both stable PMLs, regardless of the accuracy of the formulation. As stated earlier, if the parameter $\sigma_{0}$ is not properly tuned, a singularity may arise at the end layer. The convergence rate of all PMLs would be immediately affected, in the sense that the error would deviate from the reference solution for high values of $p$. 

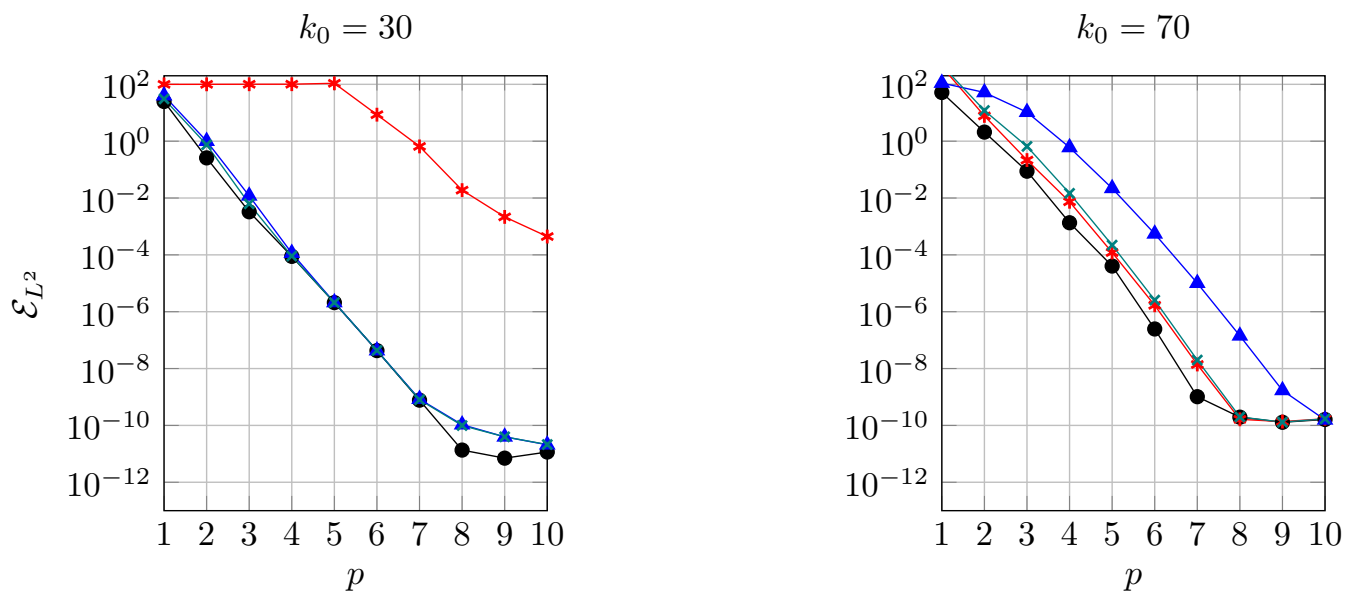

Figure 9: $L^{2}$-error (in \%) for fixed frequencies as a function of the shape function order $p$ for the classical ( $\left.\rightarrow-\right)$ ), alternative $(\multimap)$ and Lorentz (־) PMLs. Parameters: $n=3, M=0.8$ and $\sigma_{0}=4 \beta^{2}$. Reference solution $(\multimap-$ ).

\section{Cartesian PML for a flow of arbitrary direction}

Cross flows are characterized by the presence of a spatial cross derivative in the convected equation. Thereby, treating only the convective terms does not lead to a stable formulation (see Figure 1b). Dealing with cross flows is of practical interest for industrial applications, for example when a complex scatterer is present. As mentioned in Section 2.1, different choices are possible to cancel the cross derivative. Instead of the Lorentz transformation, Diaz and Joly [21] used a different space-time transformation, which is

$$
t^{*}=t+\frac{M_{x}}{\beta^{2} c_{0}} x+\frac{M_{y}}{\beta^{2} c_{0}} y, \quad x^{*}=x, \quad y^{*}=y+\frac{M_{x} M_{y}}{1-M_{x}^{2}} x .
$$

The chain rule yields the partial derivatives

$$
\partial_{x}=\partial_{x}^{*}+\frac{M_{x} M_{y}}{1-M_{x}^{2}} \partial_{y}^{*}+\frac{M_{x}}{\beta^{2} c_{0}} \partial_{t}^{*}, \quad \partial_{y}=\partial_{y}^{*}+\frac{M_{y}}{\beta^{2} c_{0}} \partial_{t}^{*}, \quad \partial_{t}=\partial_{t}^{*} .
$$

In the frequency domain, it leads to the modified equation in the transformed space

$$
\left(1-M_{x}^{2}\right) \frac{\partial^{2} \widetilde{\varphi^{*}}}{\partial x^{* 2}}+\left(1-\frac{M_{y}^{2}}{1-M_{x}^{2}}\right) \frac{\partial^{2} \widetilde{\varphi^{*}}}{\partial y^{* 2}}+\frac{k_{0}^{2}}{\beta^{2}} \widetilde{\varphi^{*}}=-f^{*},
$$

which differs from a Helmholtz-type equation. It is the natural extension of the alternative model from Section 3.3 when $M_{y} \neq 0$. The transformation relies on the analysis of the dispersion relation and does not 'stretch' the physical space. The slowness diagram describes an ellipse, whose principal axes are the $(x, y)$ directions. For that reason, the resulting PML is stable, but the absorption might not be equally effective in the $x$ - and $y$-directions. In order to avoid a jump condition issue at the PML interface, we follow the stabilization procedure explained in Figure 2, which is designed for the entire domain $\Omega$.

\subsection{Point source in free field - weak formulations}

In this example, we consider the acoustic radiation of a point source in a square domain. The problem is specified in Figure 10. We remind that we impose a homogeneous Neumann condition on the outer boundary. We do not apply any specific treatment in the PML corner regions and simply let $\gamma_{x} \neq 0$ and $\gamma_{y} \neq 0$.

The derivation of the weak formulation is similar to Section 3.2, but the calculations are more complex. It can be written as

$$
\left\{\begin{array}{l}
\text { Find } \varphi \in H^{1}(\Omega) \text { such that, } \forall \psi \in H^{1}(\Omega) \\
\int_{\Omega}\left(\frac{\gamma_{y}}{\gamma_{x}} \partial_{x^{\prime}}^{-1} \varphi \overline{\partial_{x^{\prime}}^{-1} \psi}+\frac{\gamma_{x}}{\gamma_{y}} \partial_{y^{\prime}}^{-1} \varphi \overline{\partial_{y^{\prime}}^{-1} \psi}-\frac{k_{0}^{2}}{\beta^{2}} \gamma_{x} \gamma_{y} \varphi \bar{\psi}\right) d \Omega=\int_{\Omega} f \bar{\psi} d \Omega
\end{array}\right.
$$




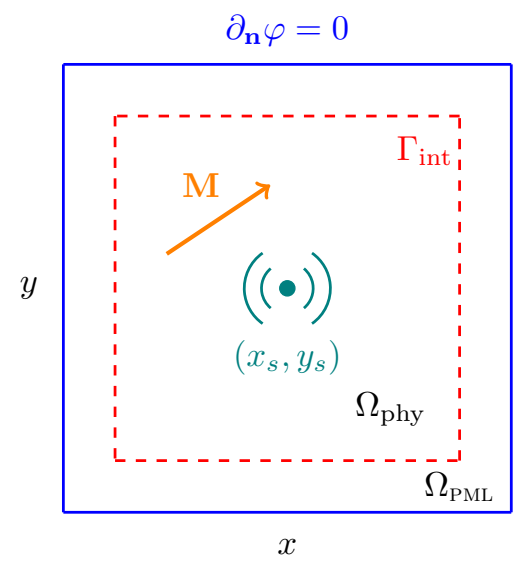

Figure 10: Sketch of the numerical case: 2D point source radiation in a cross flow with straight boundaries. The global domain $\Omega$ is the open set such that $\bar{\Omega}=\overline{\Omega_{\mathrm{phy}}} \cup \overline{\Omega_{\mathrm{PML}}}$. It is delimited by respectively $L$ and $L_{\mathrm{PML}}$.

where the notations $\partial_{x^{\prime}}^{-1}$ and $\partial_{y^{\prime}}^{-1}$ stress that the operators should be understood as inverse operators to the physical variables. From Lorentz's transformation (2.5), we can switch the role of the partial derivatives and obtain

$$
\begin{aligned}
& \partial_{x^{\prime}}^{-1}=\beta\left(\left(1+\frac{M_{y}^{2}}{\beta(1+\beta)}\right) \partial_{x}-\frac{M_{x} M_{y}}{\beta(1+\beta)} \partial_{y}-\frac{i k_{0} M_{x}}{\beta^{2}}\right), \\
& \partial_{y^{\prime}}^{-1}=\beta\left(\left(1+\frac{M_{x}^{2}}{\beta(1+\beta)}\right) \partial_{y}-\frac{M_{x} M_{y}}{\beta(1+\beta)} \partial_{x}-\frac{i k_{0} M_{y}}{\beta^{2}}\right) .
\end{aligned}
$$

In practice, the inverse transformation is written as is in the finite element routine, and there is no need to develop the stabilized model explicitly. We detail in the next Section how to write a general equation in terms of matrix transformations. For comparison, the alternative inverse transformation associated to (4.1) takes a simpler form

$$
\partial_{x^{*}}^{-1}=\partial_{x}-\frac{M_{x} M_{y}}{1-M_{x}^{2}} \partial_{y^{*}}^{-1}-\frac{i k_{0} M_{x}}{\beta^{2}}, \quad \partial_{y^{*}}^{-1}=\partial_{y}-\frac{i k_{0} M_{y}}{\beta^{2}} .
$$

The associated weak formulation is built from domain equation (4.3) and reverted to the physical variables by applying the operators $\partial_{x^{*}}^{-1}$ and $\partial_{y^{*}}^{-1}$. Intuitively, the difference with the Lorentz model lies in the way the cross derivatives are handled.

\subsection{Point source in free field - numerical setup and results}

The point source is defined as a single monopole. In free field, the analytical solution is naturally defined thanks to the transformed Lorentz variables as

$$
\varphi_{\mathrm{ex}}(x, y, \omega)=\frac{1}{\beta} \varphi_{\mathrm{ex}}^{\prime}\left(x^{\prime}, y^{\prime}, \omega^{\prime}\right) e^{i k_{0}^{\prime} M_{x} x^{\prime}} e^{i k_{0}^{\prime} M_{y} y^{\prime}}
$$

where

$$
\varphi_{\mathrm{ex}}^{\prime}\left(x^{\prime}, y^{\prime}, \omega^{\prime}\right)=-\frac{i}{4} H_{0}^{(2)}\left(k_{0}^{\prime} r^{\prime}\right) \quad r^{\prime}=\sqrt{\left(x-x_{s}\right)^{\prime 2}+\left(y-y_{s}\right)^{\prime 2}},
$$

and $H_{0}^{(2)}$ is the Hankel function of the second kind and $\boldsymbol{x}_{s}=\left(x_{s}, y_{s}\right)^{T}$ is the source position. The solution $\varphi_{\mathrm{ex}}$ is then implemented in the physical variables $(x, y, \omega)$ through the initial transformation (2.4). For the computations, we define the number of degrees of freedom per shortest wavelength as [44]

$$
d_{\lambda}=\frac{2 \pi p}{\omega h}(1-M)
$$


Unless explicitly mentioned in the numerical results, we fix the shape function order to $p=6$, the frequency to $\omega=6 \pi$ and choose a meshsize $h=0.07$. The Mach number is $M=0.8$, leading to $d_{\lambda} \approx 5.7$. The flow is defined at an angle $\theta \in[0,2 \pi]$. We surround the physical domain by two PML layers of size $h$. The PML parameter is set to $\sigma_{0}=\beta$, which is later justified by the simulations. Linear triangular elements T3 are used to generate an unstructured mesh in both $\Omega_{\text {phy }}$ and $\Omega_{\mathrm{PML}}$.

The analytical solution is singular at $\boldsymbol{x}_{s}$ and special attention is required to compute the domain $L^{2}$-error. We use the strategy suggested in [50], which consists in excluding the one-ring neighbourhood elements to the point source. In that way, the authors have shown that the usual finite element convergence properties are recovered. The relative $L^{2}$-error is defined as

$$
\mathcal{E}_{L^{2}}=100 \frac{\left\|\varphi_{\mathrm{ex}}-\varphi_{h}\right\|_{L^{2}\left(\Omega_{\rho}\right)}}{\left\|\varphi_{\mathrm{ex}}\right\|_{L^{2}\left(\Omega_{\rho}\right)}}, \quad \Omega_{\rho}=\Omega_{\mathrm{phy}} \backslash B_{\rho}\left(\boldsymbol{x}_{\boldsymbol{s}}\right),
$$

where $B_{\rho}\left(\boldsymbol{x}_{\boldsymbol{s}}\right)$ is the ball of radius $\rho$ centered at $\boldsymbol{x}_{\boldsymbol{s}}$. In the simulations, the source is set at the origin where the mesh is refined by a factor 2. Using a finer mesh close to the source allows to confine the singularity errors in a more compact region. The ball radius should be of the order of the meshsize: it is set to $\rho=2 h$.

In addition to the domain $L^{2}$-error, we measure the interface $L^{2}$-error on $\Gamma_{\text {int }}$, defined as

$$
\mathcal{E}_{L^{2}}^{I}=100 \frac{\left\|\varphi_{\mathrm{ex}}-\varphi_{h}\right\|_{L^{2}\left(\Gamma_{\mathrm{int}}\right)}}{\left\|\varphi_{\mathrm{ex}}\right\|_{L^{2}\left(\Gamma_{\mathrm{int}}\right)}}, \quad \Gamma_{\mathrm{int}}=\overline{\Omega_{\mathrm{phy}}} \cap \overline{\Omega_{\mathrm{PML}}},
$$

and the computational mesh is constrained to have nodes along the control line $\Gamma_{\text {int }}$.

The real part of the numerical solution is shown in Figure 11 for the three models at $\theta=\pi / 4$. The local interface error is plotted along $\Gamma_{\text {int }}$ in Figure 12. This immediately highlights a stronger symmetry property of the Lorentz stabilization. The error pattern repeats itself after a $180^{\circ}$ rotation for the alternative model, while this reduces to $90^{\circ}$ for Lorentz's model. Note that this property has been observed for both symmetric and non-symmetric meshes.

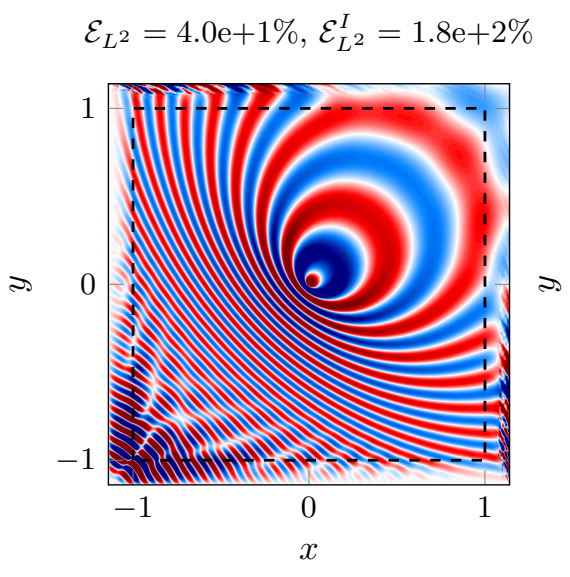

(a) Classical

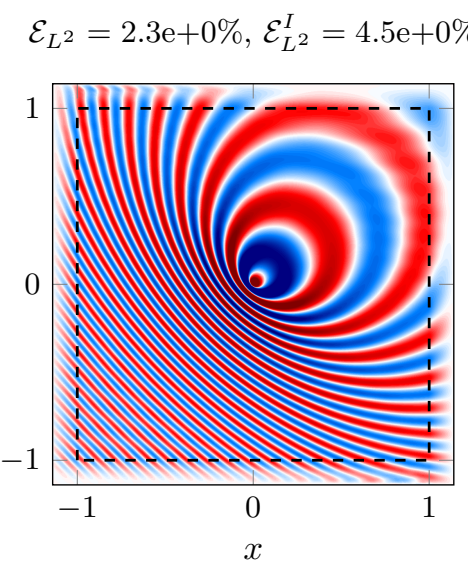

(b) Alternative

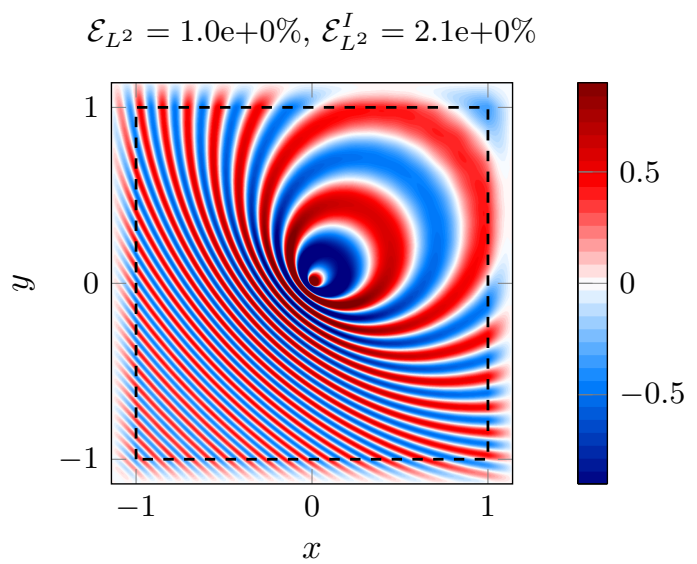

(c) Lorentz

Figure 11: Real part of the numerical solution at $\omega=6 \pi, M=0.8$ and $\theta=\pi / 4$ for the three PML models with $\sigma_{0}=\beta$.

The same conclusion follows in Figure 13 where the flow angle is varied in the range $[0,2 \pi]$. With Lorentz's model, the error is always maximal when $\theta=\pi / 4 \pm \pi / 2$ (when the flow is oriented towards the corner). This accuracy difference between the two stable formulations is most likely linked to the equations in the transformed space, respectively (4.3) and (2.6) for the alternative and Lorentz models. Note that if the flow orientation is orthogonal to one of the PML layers, both models are equivalent.

Since the Lorentz PML acts on a Helmholtz problem, it should inherit from the same discrete properties. Because the wave propagates in free field, we could choose by extension $\sigma_{0} \approx \beta c_{0}$ as recommended by Bermúdez et al. [7]. This choice is confirmed in Figure 14, where the domain $L^{2}$-error is shown as a function of the normalized parameter $\sigma_{0} / \beta$. One remarks that, for a small parameter range, the classical PML gives a solution with a reasonable accuracy. This hides the unstable nature of the formulation, a slight variation 


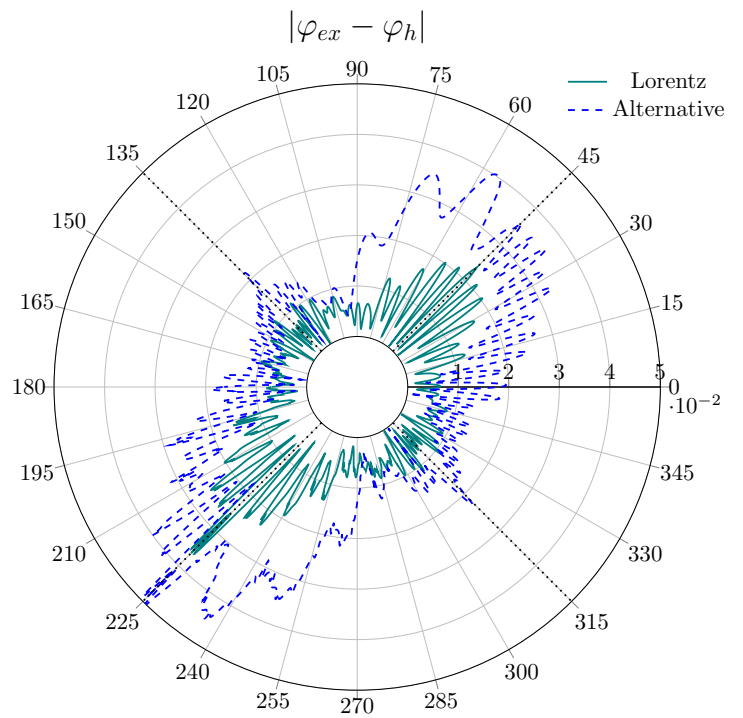

Figure 12: Polar diagram of the local error along the PML interface Figure 13: Domain and interface $L^{2}$-errors (in \%) as a associated to Figures $11 \mathrm{~b}$ and 11c.
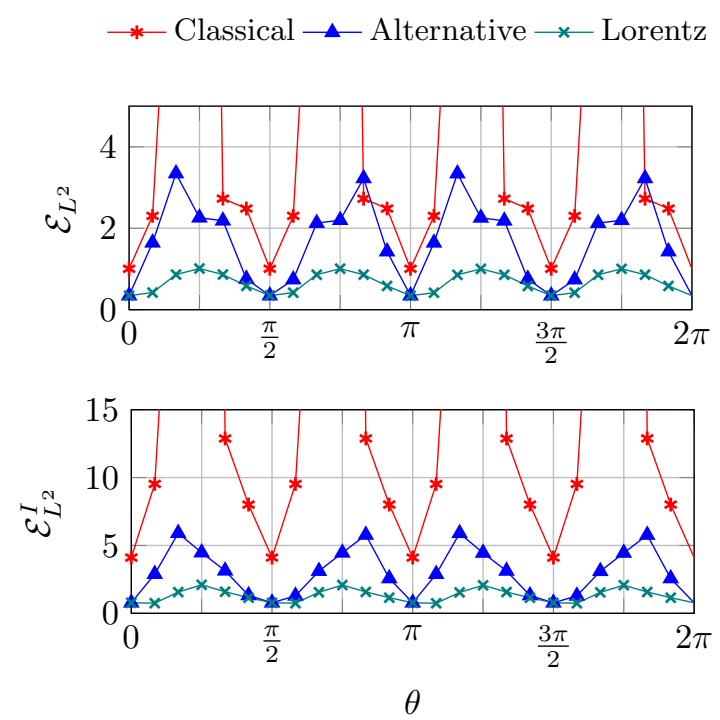

function of the flow angle $\theta$ for $M=0.8$ with $\sigma_{0}=\beta$.

of $\sigma_{0}$ would completely deteriorate the solution. In Figure 15, both unstable and stabilized models converge towards the physical solution when the number of PML layers increases. This property has been proven in [16]. One must be careful when using the unstable formulation. Unlike in time-domain acoustics, it is possible to incidentally obtain a PML of rather good accuracy if the instabilities are not 'too strong'. A similar conclusion was drawn for an unstable PML applied to the Schrödinger equation, where the phenomenon is referred to as conditional stability [51].

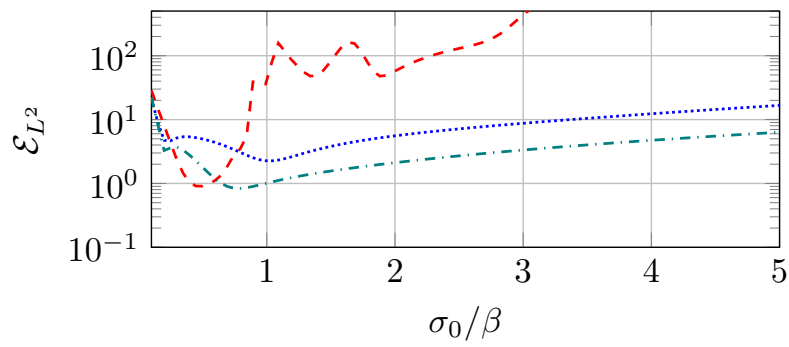

(a) $\theta=\pi / 4$.

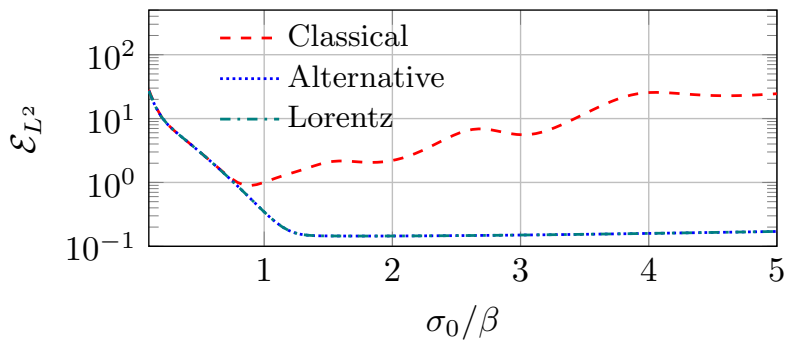

(b) $\theta=0$.

Figure 14: $L^{2}$-error (in \%) with the unbounded PML profile (2.9) as a function of $\sigma_{0}$ for $M=0.8$ and $\omega=6 \pi$.

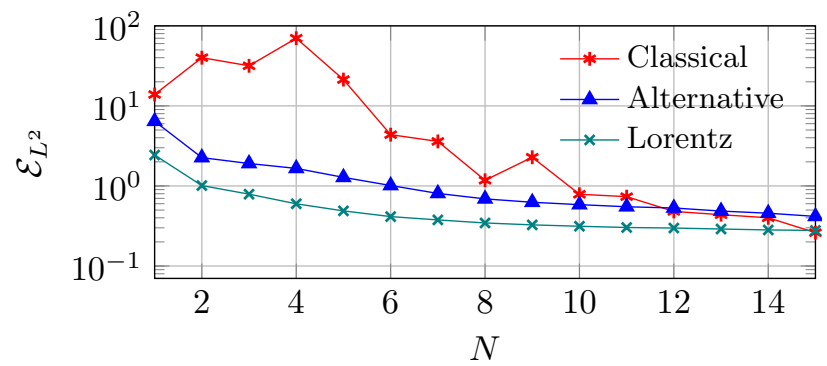

Figure 15: Influence of the number of PML layers $N$ on the $L^{2}$-error (in \%) at $\theta=\pi / 4, M=0.8, \omega=6 \pi$ and $\sigma_{0}=\beta$. 
We would like to study the robustness of the stable PML with respect to the Mach number. While doing so it is helpful to maintain an equivalent discretization accuracy when $M$ varies. We resort to the $a$ priori error indicator proposed in [52], which adjusts the order across the mesh so as to achieve a given, user defined $L^{2}$-error target accuracy $\mathcal{E}_{T}$. In practice, the edge orders are first determined based on a 1D error indicator, which accounts for the local in-flow dispersion relation properties and possible edge curvature. In a second step, the element interior (directional) orders are assigned through a set of simple element-type dependent conformity rules. Note that this approach does not account for the pollution effect. The orders are here defined to be in the range $p \in[1,15]$. To serve as a reference, we measure the relative $L^{2}$-error between the exact solution and its $L^{2}$-projection $\mathcal{P} \varphi_{\text {ex }}$ onto the high-order finite element space,

$$
\mathcal{E}_{L^{2}}^{\mathrm{best}}=100 \frac{\left\|\mathcal{P} \varphi_{\mathrm{ex}}-\varphi_{\mathrm{ex}}\right\|_{L^{2}\left(\Omega_{\rho}\right)}}{\left\|\varphi_{\mathrm{ex}}\right\|_{L^{2}\left(\Omega_{\rho}\right)}}
$$

This error (also referred to as best interpolation error) corresponds to the best numerical solution that can be achieved in the physical domain by a given approximation basis, regardless of the chosen formulation (see e.g. [53, section 5]).

Figure 16 presents the dependence of the domain $L^{2}$-error when the Mach number varies, for $\mathcal{E}_{T}=3 \%$ and $\mathcal{E}_{T}=0.5 \%$ as target errors. Results are shown for $\theta=0$ and $\theta=\pi / 4$. The usual PML shows decent performance but deteriorates when the flow is strong or not aligned with the PML. When $\theta=0$, both approaches exhibit similar accuracy except for $M=0.9$. When $\theta=\pi / 4$, the instability of the classical model is more significant for high values of $M$ (from $M \approx 0.6$ ) since the non-zero cross flow strengthens the instability. It is worth mentioning that the best $L^{2}$-error remains constant on the full Mach number range, which indicates that the a priori error indicator appropriately selects the order distribution for each configuration, which varies significantly between low and high Mach number values.

When $\theta=\pi / 4$, the convergence rate of the stable PML seems slightly affected for high Mach numbers. Numerical experiments have shown that it is mostly imputable to the PML reflections, which may come from a corner effect.

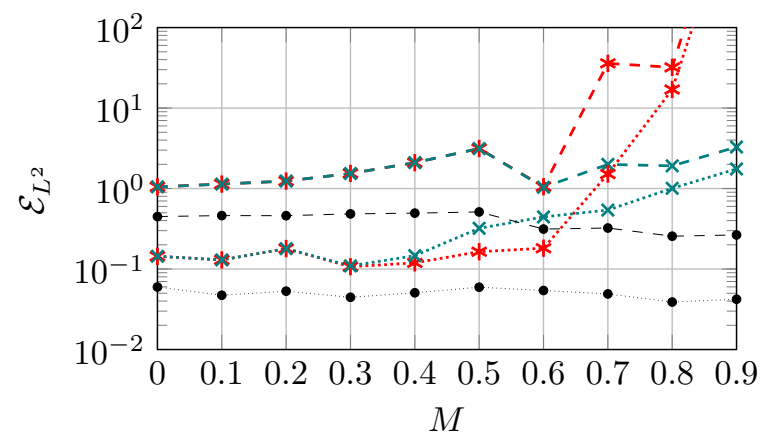

(a) $\theta=+\pi / 4$.

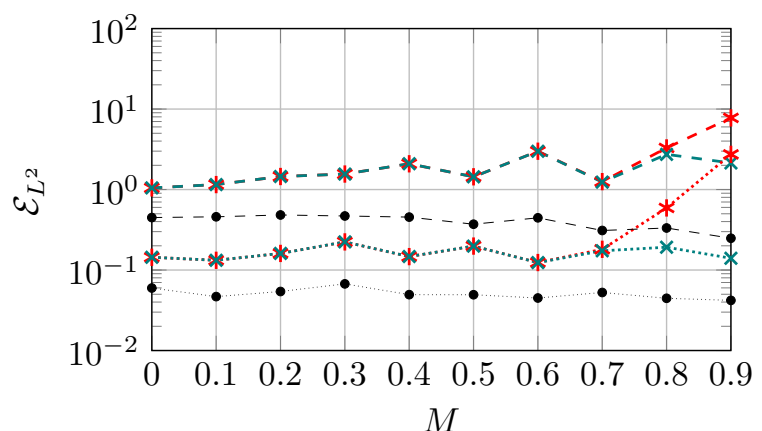

(b) $\theta=0$.

Figure 16: $L^{2}$-error (in \%) as a function of the Mach number for two target errors with $\sigma_{0}=\beta$. Legend: classical $-\mathcal{E}_{T}=3 \%$ $(-*-)$, Lorentz $-\mathcal{E}_{T}=3 \%(-\star-)$, classical $-\mathcal{E}_{T}=0.5 \%(\cdots * \cdots)$, Lorentz $-\mathcal{E}_{T}=0.5 \%(\cdots \cdot \cdots \cdots)$, best interpolation for $\mathcal{E}_{T}=3 \%$ $(-\bullet-)$ and $\mathcal{E}_{T}=0.5 \%(\cdots \bullet \cdots)$.

\section{Stable PML of arbitrary convex shape}

We now extend the approach to a PML domain of arbitrary convex shape. The basic idea consists in formulating the PML in curvilinear coordinates as described by Collino and Monk [4], but in the Lorentz space. Then, we apply the inverse Lorentz transformation to obtain the stabilized PML model. In curvilinear coordinates, it is possible to recast the PML formulation in Cartesian coordinates as follows

$$
\nabla_{\boldsymbol{x}} \cdot\left(\boldsymbol{\Lambda}_{\mathrm{PML}} \nabla_{\boldsymbol{x}} \widetilde{\varphi}\right)+\alpha_{\mathrm{PML}} k_{0}^{2} \widetilde{\varphi}=-f
$$


where

$$
\boldsymbol{\Lambda}_{\mathrm{PML}}=\alpha_{\mathrm{PML}} \boldsymbol{J}_{\mathrm{PML}}^{-1} \boldsymbol{J}_{\mathrm{PML}}^{-T}, \quad \alpha_{\mathrm{PML}}=\operatorname{det} \boldsymbol{J}_{\mathrm{PML}}, \quad \boldsymbol{J}_{\mathrm{PML}}=\frac{\partial \widetilde{\boldsymbol{x}}}{\partial \boldsymbol{x}} .
$$

The matrix $\boldsymbol{\Lambda}_{\mathrm{PML}}$ is symmetric and couples the metric of $\Omega_{\mathrm{PML}}$ with the complex stretching parameters. It encodes all the information related to the PML. For the following development, we write

$$
\boldsymbol{\Lambda}_{\mathrm{PML}}=\left(\begin{array}{cc}
\Lambda_{11} & \Lambda_{12} \\
\Lambda_{21} & \Lambda_{22}
\end{array}\right), \quad \Lambda_{12}=\Lambda_{21}
$$

If for instance the PML domain is rectangular, the entries are $\Lambda_{11}=\gamma_{y} / \gamma_{x}, \Lambda_{22}=\gamma_{x} / \gamma_{y}, \Lambda_{12}=\Lambda_{21}=0$. Note that one could use a more complicated tensor that accounts for the discrete properties of the mesh such as, but not limited to, the locally-conformal PML technique [54, 55].

Independently of the technique used, we shall apply the inverse Lorentz transform to the Helmholtz-PML equation in the Lorentz space

$$
\nabla_{\boldsymbol{x}^{\prime}}^{-1} \cdot\left(\boldsymbol{\Lambda}_{\mathrm{PML}} \nabla_{\boldsymbol{x}^{\prime}}^{-1} \widetilde{\varphi}\right)+\alpha_{\mathrm{PML}}{k_{0}^{\prime}}^{2} \widetilde{\varphi}=-f, \quad k_{0}^{\prime}=\frac{k_{0}}{\beta},
$$

where $\nabla_{\boldsymbol{x}^{\prime}}^{-1}$ is the modified gradient from the inverse Lorentz transformation

$$
\nabla_{\boldsymbol{x}^{\prime}}^{-1}=\boldsymbol{L}^{-1} \nabla_{\boldsymbol{x}}-\frac{i k_{0}}{\beta} \boldsymbol{M}, \quad \boldsymbol{L}_{i j}=\delta_{i j}+\frac{M_{x_{i}} M_{x_{j}}}{\beta(1+\beta)}, \quad \delta_{i j}= \begin{cases}1, & \text { if } i=j, \\ 0, & \text { if } i \neq j .\end{cases}
$$

A simple calculation shows that $\operatorname{det}(\boldsymbol{L})=1 / \beta$. We can now develop equation (5.4) to get a general stabilized model in free field

$$
\begin{aligned}
\boldsymbol{L}^{-1} \nabla_{\boldsymbol{x}} \cdot\left(\boldsymbol{\Lambda}_{\mathrm{PML}} \boldsymbol{L}^{-1} \nabla_{\boldsymbol{x}} \widetilde{\varphi}\right) & -\frac{i k_{0}}{\beta} \boldsymbol{M} \cdot\left(\boldsymbol{\Lambda}_{\mathrm{PML}} \boldsymbol{L}^{-1} \nabla_{\boldsymbol{x}} \widetilde{\varphi}\right)-\frac{k_{0}^{2}}{\beta^{2}}\left[\boldsymbol{M} \cdot\left(\boldsymbol{\Lambda}_{\mathrm{PML}} \boldsymbol{M}\right)-\alpha_{\mathrm{PML}}\right] \widetilde{\varphi} \\
& -\frac{i k_{0}}{\beta}\left(\boldsymbol{L}^{-1} \nabla_{\boldsymbol{x}}\right) \cdot\left(\boldsymbol{\Lambda}_{\mathrm{PML}} \boldsymbol{M} \widetilde{\varphi}\right)=-f .
\end{aligned}
$$

By using the product rule on the last term of the left-hand side, and the symmetry of $\boldsymbol{L}^{-1}$ and $\boldsymbol{\Lambda}_{\mathrm{PML}}$, the model is recast as

$$
\begin{aligned}
\boldsymbol{L}^{-1} \nabla_{\boldsymbol{x}} \cdot\left(\boldsymbol{\Lambda}_{\mathrm{PML}} \boldsymbol{L}^{-1} \nabla_{\boldsymbol{x}} \widetilde{\varphi}\right) & -2 \frac{i k_{0}}{\beta}\left(\boldsymbol{L}^{-1} \boldsymbol{\Lambda}_{\mathrm{PML}} \boldsymbol{M}\right) \cdot \nabla_{\boldsymbol{x}} \widetilde{\varphi}-\frac{k_{0}^{2}}{\beta^{2}}\left[\boldsymbol{M} \cdot\left(\boldsymbol{\Lambda}_{\mathrm{PML}} \boldsymbol{M}\right)-\alpha_{\mathrm{PML}}\right] \widetilde{\varphi} \\
& -\frac{i k_{0}}{\beta} \boldsymbol{M} \cdot\left[\left(\boldsymbol{L}^{-1} \nabla_{\boldsymbol{x}}\right) \cdot \boldsymbol{\Lambda}_{\mathrm{PML}}\right] \widetilde{\varphi}=-f .
\end{aligned}
$$

where $\left(\boldsymbol{L}^{-1} \nabla_{\boldsymbol{x}}\right) \cdot \boldsymbol{\Lambda}_{\mathrm{PML}}$ is a column vector of components

$$
\left[\left(\boldsymbol{L}^{-1} \nabla_{\boldsymbol{x}}\right) \cdot \boldsymbol{\Lambda}_{\mathrm{PML}}\right]_{i}=\sum_{j, k} L_{j k}^{-1} \partial_{x_{k}}\left(\Lambda_{j i}\right), \quad i=\{1,2\} .
$$

Thanks to the useful relation $\boldsymbol{L}^{-1} \boldsymbol{M}=\beta \boldsymbol{M}$ [32], we may recognize a generalization of model (3.5). The weak formulation is obtained similarly by starting from Equation (5.4). This approach leads by construction to a symmetric bilinear formulation. In terms of PML Jacobian matrix $[3,56]$, we may express

$$
\left\{\begin{array}{l}
\text { Find } \varphi \in H^{1}(\Omega) \text { such that, } \forall \psi \in H^{1}(\Omega), \\
\int_{\Omega}\left[\left(\boldsymbol{J}_{\mathrm{PML}}^{-T} \nabla_{\boldsymbol{x}^{\prime}}^{-1} \varphi\right) \cdot\left(\boldsymbol{J}_{\mathrm{PML}}^{-T} \overline{\nabla_{\boldsymbol{x}^{\prime}}^{-1} \psi}\right)-\frac{k_{0}^{2}}{\beta^{2}} \varphi \bar{\psi}\right] \operatorname{det}\left(\boldsymbol{J}_{\mathrm{PML}} \boldsymbol{L}\right) d \Omega=\int_{\Omega} f \bar{\psi} \operatorname{det}(\boldsymbol{L}) d \Omega .
\end{array}\right.
$$

Since $\operatorname{det}(\boldsymbol{L}) \neq 0$, it can here be simplified. This term is however important to recover non-homogeneous boundary conditions in the physical variables. Let us develop the integrand related to the inverse transfor- 
mation

$$
\begin{aligned}
& \left(\boldsymbol{J}_{\mathrm{PML}}^{-T} \nabla_{\boldsymbol{x}^{\prime}}^{-1} \varphi\right) \cdot\left(\boldsymbol{J}_{\mathrm{PML}}^{-T} \overline{\nabla_{\boldsymbol{x}^{\prime}}^{-1} \psi}\right)=\left(\left(\boldsymbol{J}_{\mathrm{PML}} \boldsymbol{L}\right)^{-T} \nabla_{\boldsymbol{x}} \varphi-\frac{i k_{0}}{\beta}\left(\boldsymbol{J}_{\mathrm{PML}}^{-T} \boldsymbol{M}\right) \varphi\right) \cdot\left(\left(\boldsymbol{J}_{\mathrm{PML}} \boldsymbol{L}\right)^{-T} \nabla_{\boldsymbol{x}} \bar{\psi}+\frac{i k_{0}}{\beta}\left(\boldsymbol{J}_{\mathrm{PML}}^{-T} \boldsymbol{M}\right) \bar{\psi}\right) \\
& =\left(\boldsymbol{J}_{\mathrm{PML}} \boldsymbol{L}\right)^{-T} \nabla_{\boldsymbol{x}} \varphi \cdot\left(\boldsymbol{J}_{\mathrm{PML}} \boldsymbol{L}\right)^{-T} \nabla_{\boldsymbol{x}} \bar{\psi}+\frac{k_{0}^{2}}{\beta^{2}}\left(\boldsymbol{J}_{\mathrm{PML}}^{-T} \boldsymbol{M}\right) \varphi \cdot\left(\boldsymbol{J}_{\mathrm{PML}}^{-T} \boldsymbol{M}\right) \bar{\psi} \\
& +\frac{i k_{0}}{\beta}\left(\boldsymbol{J}_{\mathrm{PML}} \boldsymbol{L}\right)^{-T} \nabla_{\boldsymbol{x}} \varphi \cdot\left(\boldsymbol{J}_{\mathrm{PML}}^{-T} \boldsymbol{M}\right) \bar{\psi}-\frac{i k_{0}}{\beta}\left(\boldsymbol{J}_{\mathrm{PML}}^{-T} \boldsymbol{M}\right) \varphi \cdot\left(\boldsymbol{J}_{\mathrm{PML}} \boldsymbol{L}\right)^{-T} \nabla_{\boldsymbol{x}} \bar{\psi}
\end{aligned}
$$

Each term is a $2 \times 1$ vector and resemble the uni-axial situation (3.15). The expanded weak statement directly follows. To sum up, the method consists in three steps:

1. set up the bilinear form associated to the modified Helmholtz equation in the Lorentz space,

2. apply the curvilinear PML through the Jacobian matrix $\boldsymbol{J}_{\mathrm{PML}}$ or the matrix $\boldsymbol{\Lambda}_{\mathrm{PML}}$,

3. modify the definition of the usual gradient thanks to the inverse Lorentz transformation.

This process only involves metric transformations, the rest being automatically handled by the finite element code. It is also worth emphasizing that although the construction of the stabilized formulation is based on the entire domain $\Omega$, it does not change the convected Helmholtz equation in the physical domain. This implies that one could potentially solve a non-uniform flow problem in $\Omega_{\text {phy }}$, such as the linearized potential equation [52], and use the formulation (5.9) only in $\Omega_{\mathrm{PML}}$. One must ensure however that the flow properties do not vary on the PML interface.

\subsection{Illustration for a circular $P M L$}

For a circular boundary, the PML Jacobian matrix in polar coordinates $(r, \phi)$ is

$$
\boldsymbol{J}_{\mathrm{PML}}^{-T}=\left(\begin{array}{cc}
\cos \phi / \gamma & \sin \phi / \gamma \\
-\sin \phi / \hat{\gamma} & \cos \phi / \hat{\gamma}
\end{array}\right), \quad \hat{\gamma}=1-\frac{i}{r k_{0}} \zeta(r), \quad \gamma=1-\frac{i}{k_{0}} \sigma(r),
$$

where $\zeta$ and $\sigma$ are defined in (2.9) for the radial variable $r \in\left[R, R_{\mathrm{PML}}\right]$. For the illustrations, we choose a flow magnitude $M=0.8$ and a running frequency $\omega=20 \pi$. The mesh size is $h=0.03$ and the shape function orders are given by the anisotropic order assignement with an error target $\mathcal{E}_{T}=0.5 \%$ [52]. The physical domain is composed of T3 elements. Two PML layers of Q4 elements are extruded with extrusion length $h$ from the physical domain, of size $R=1$. Note that such a hybrid mesh is not a requirement for the proposed method. The PML parameter is $\sigma_{0}=\beta$, and the mesh is refined by a factor 2 around the origin.

We present in Figure 17 the result of the stabilization process for a multiple point sources configuration. The setup can be assimilated to a lateral quadrupole: four equally spaced monopoles with alternating phase are positioned at the corners of a square of size $\delta=5 \mathrm{e}-3$. Note that the frequency in the physical domain spans from $\omega_{\min }=\omega /(1+M)$ to $\omega_{\max }=\omega /(1-M)$. As expected the flow has also a large impact on the radiation pattern of the quadrupole. The four directivity lobes are not symmetric anymore, and the sound is refracted upstream. Interestingly, the upstream silence cone is found to be narrower, while the one downstream is significantly enlarged.

The classical PML formulation is affected by the instability of the upstream wave and does not yield an accurate solution for this configuration, with an error measured at $\mathcal{E}_{L^{2}}=35 \%$, as shown in Figure 17 a. The Lorentz formulation on the other hand, presented in Figure $17 \mathrm{~b}$ is well behaved, and delivers a solution with an error close to the target accuracy. Note that the typical shape function order for this approximation, as determined by the a priori error indicator is $p=7$. The full discrete model involves approximately 350000 degrees of freedom and 17 million non-zeros entries, of which approximately $6 \%$ originate from the PML.

Figure 18a presents the domain and interface errors when the flow angle varies, for the monopole and lateral quadrupole configurations. By contrast to the Cartesian case, the error is almost independent of the flow direction, and the behaviour does not appear to be altered by the complexity of the source. Note that a slightly lower error is observed for the quadrupole at $\theta=0 \pm \pi / 2$, when the flow is oriented along the non-convected quadrupole zone of silence. The formulation is robust with respect to the Mach number in the sense that the error follows the trend of the best interpolation, which holds even for high Mach numbers. 


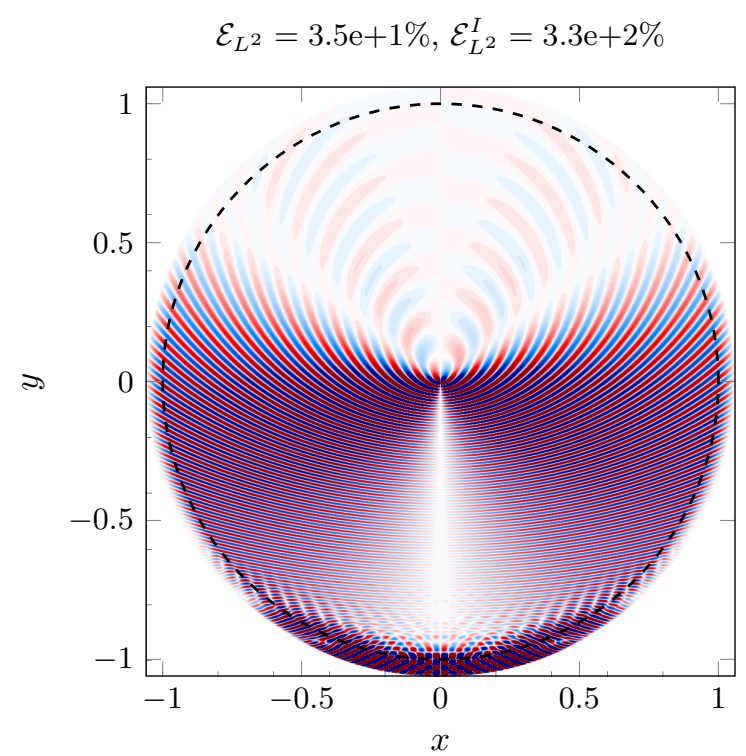

(a) Classical

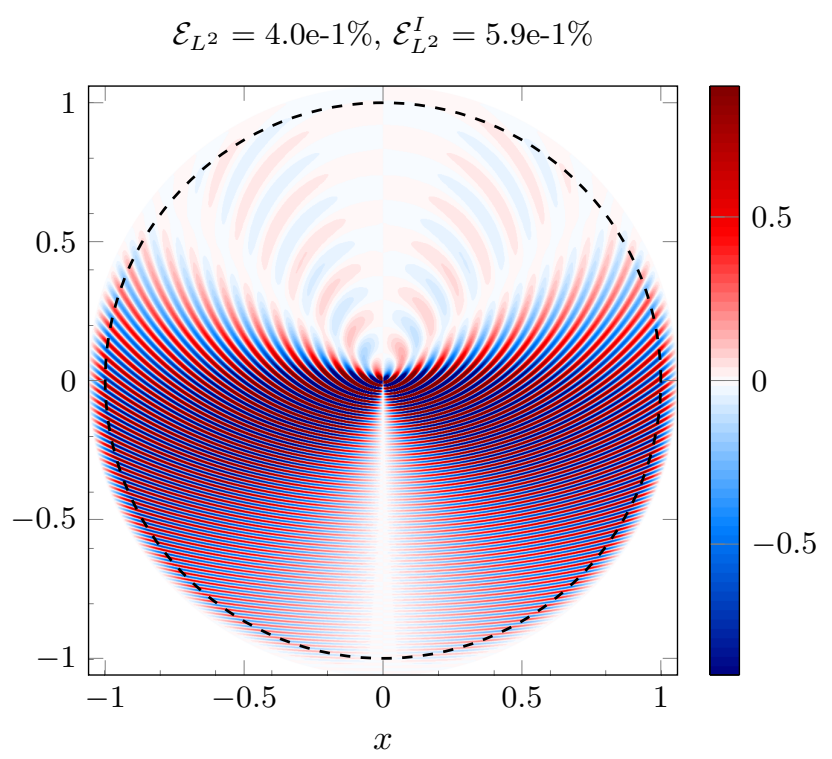

(b) Lorentz

Figure 17: Real part of the numerical solution at $\omega=20 \pi, M=0.8$ and $\theta=\pi / 2$ for the unstable and stable PML models with $\sigma_{0}=\beta$.

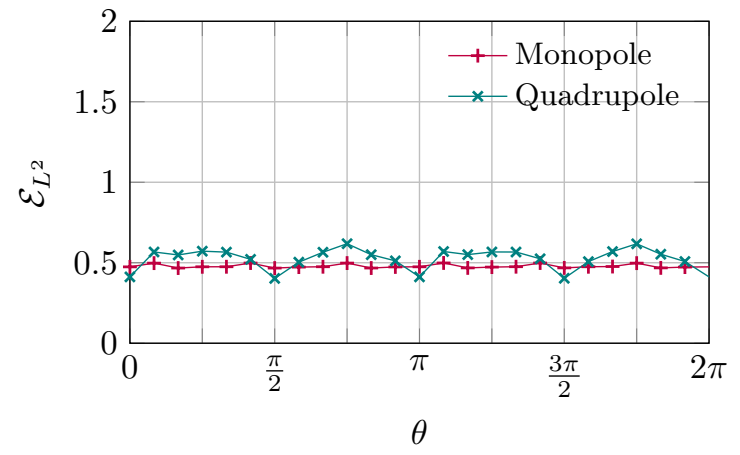

(a) $M=0.8, \mathcal{E}_{T}=0.5 \%$.

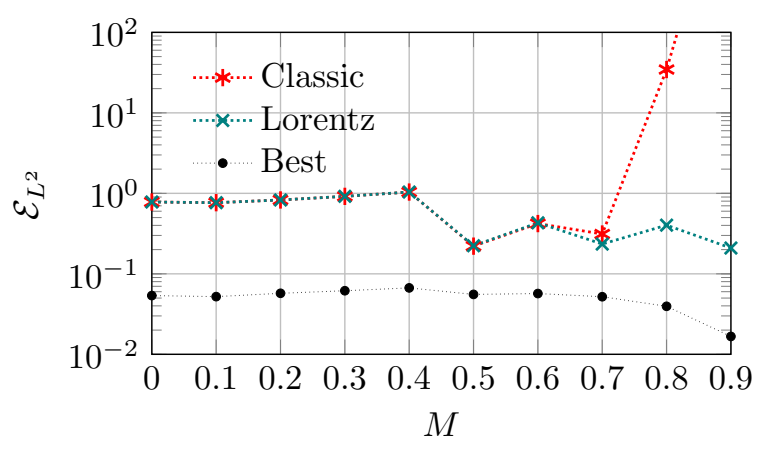

(b) Quadrupole case: $\mathcal{E}_{T}=0.5 \%, \theta=\pi / 2$.

Figure 18: $L^{2}$-error (in \%) for the circular PML as a function of the flow angle and Mach number.

\subsection{Additional remarks for more complex problems}

Extensions to the Lorentz transformation could be investigated if the flow has to be non-uniform on the PML interface. Taylor [27] proposed an extension to low Mach number potential flows, which was further proven in [31] to be valid for all frequencies. If novel instabilities come out, it would be interesting to analyze the impact of the Taylor-Lorentz transformation over the Lorentz one. Hu [15] noticed that for a certain category of non-uniform flows, the modes follow a peculiar pattern on the dispersion relation diagram, and that convective instabilities could still be handled in a similar fashion.

The present work could not a priori be extended to the linearized Euler equations for cross flows, because there is currently no space-time transformation that can treat the instabilities simultaneously for acoustic, vorticity and entropy waves [23]. Lorentz transformation is no exception to it, but seems nevertheless to be applicable if the linearized Euler equations are written in terms of momentum instead of velocity perturbations $[20]$. 


\section{Conclusion}

Following the pioneer work from Bécache et al. $[16]$ and $\mathrm{Hu}[14,15]$, we have proposed a practical procedure to design a stable curvilinear PML for the convected Helmholtz equation with a flow of arbitrary orientation. The heart of the method lies in the Lorentz transformation. By reducing the convected Helmholtz equation into a classical Helmholtz equation, the proposed Lorentz PML is clearly stable and shares the same features as usual PMLs. The analysis was conducted in two-dimensions but the extension to the three-dimensional case is direct. Numerical investigations pointed out the stability limit of the classical PML and justified the use of Lorentz's PML over existing stable formulations in terms of numerical accuracy. The efficiency of the method was illustrated for both modal and free field problems. Extension to the linearized Euler equations and application as a transmission condition for domain decomposition will be investigated in the future.

\section{Acknowledgments}

This work was performed as part of CIFRE contract No. 2018/1845 funded by Siemens Industry Software SAS and Association Nationale de la Recherche et de la Technologie (ANRT). This research was also funded in part through the ARC grant for Concerted Research Actions (ARC WAVES 15/19-03), financed by the Wallonia-Brussels Federation of Belgium. The authors would like to thank Prof. Gwénaël Gabard for his helpful guidance on the Lorentz transformation. We thank the anonymous reviewers for their careful reading of our manuscript and their insightful comments and suggestions.

\section{Appendix A. Selection of the PML parameter for high-order FEM}

In this appendix, we discuss the influence of the PML parameter $\sigma_{0}$ on the convergence rate of the $p$-FEM. Let us consider the duct case from Section 3.1 and set $M=0$ for simplicity. We use the third mode at the frequency $k_{0}=50$. Four equally-spaced elements are used to mesh the PML domain along the $x$-direction. To begin with, Figure A.19a depicts the modulus of the exact PML solution $\widetilde{\varphi}$ in one-dimension, which is given by equation (2.11). It is clear that the induced decay function $|\widetilde{\varphi}|$ has a singularity at $x=L_{\mathrm{PML}}$ when $\sigma_{0}<V_{\mathrm{p}}$, is polynomial of order $n$ when $\sigma_{0}=n V_{\mathrm{p}}\left(|\widetilde{\varphi}|\right.$ is linear when $\left.\sigma_{0}=V_{\mathrm{p}}\right)$ and otherwise, gains in regularity as $\sigma_{0}>V_{\mathrm{p}}$ grows. We recall that since the absorbing function $\sigma$ is unbounded, the PML is reflectionless at the continuous level. Note that the homogeneous Neumann boundary condition imposed at $x=L_{\mathrm{PML}}$ (see Figure 3 ) is not verified when $\sigma_{0} \leq V_{\mathrm{p}}$, because a homogeneous Dirichlet condition is automatically enforced by the use of the unbounded absorbing function [8]. We have conducted additional experiments and observed little numerical impact due to the end layer boundary condition.

Figure A.19b presents the associated $L^{2}$-error in $\Omega_{\text {phy }}$ for an increasing order $p$ for the values of $\sigma_{0}$ depicted in Figure A.19a. It may be observed that the convergence rate is algebraic for $\sigma_{0}=V_{\mathrm{p}} / 2$ and only partially exponential for $\sigma_{0}=3 V_{\mathrm{p}} / 2$, due to the lack of regularity of the solution at the layer end. On the other hand, the $p$-convergence is optimal for $\sigma_{0}=V_{\mathrm{p}}$ and nearly optimal for $\sigma_{0}=5 V_{\mathrm{p}} / 2$ respectively. Interestingly, the $p$-convergence is exponential for $\sigma_{0}=20 V_{\mathrm{p}}$ but the error is orders of magnitude higher than the reference solution.

In order to further interpret these results, the relative $L^{2}$-error and the best $L^{2}$-error defined in equation (4.12) are recorded as a function of $\sigma_{0}$ for different orders $p$, this time not in $\Omega_{\text {phy }}$, but in $\Omega_{\mathrm{PML}}$ only. This is shown in Figure A.20. The relative $L^{2}$-error fits the best interpolation, which confirms that the maximal accuracy is achieved at a given order $p$. As expected, the interpolation (of the non oscillatory part) of the solution is exact when the ratio $\sigma_{0} / V_{\mathrm{p}}$ is an integer. However if $\sigma_{0}$ is chosen too large, the fast decay of $\widetilde{\varphi}$ cannot be fully captured by the PML mesh and the error increases. Most importantly, Figure A.20 highlights that the regularity of $|\widetilde{\varphi}|$ at $x=L_{\mathrm{PML}}$ governs the potential gain in accuracy when increasing the polynomial order, which confirms previous observations. It is worth mentioning that the same results were obtained when removing the $y$-dependency, hence the performance of the PML can be analyzed through a simple 1D interpolation problem. In short, we can affirm that there exists an optimal $\sigma_{0}$ regime where the interpolation error of the one-dimensional decay function (the non-oscillatory part) is small enough such that the discrete PML is efficient. It is attained from roughly $\sigma_{0}>V_{p}$. The regime is referred to as sub-optimal when $\sigma_{0}<V_{p}$ and sup-optimal when $\sigma_{0} \gg V_{p}[55]$. 


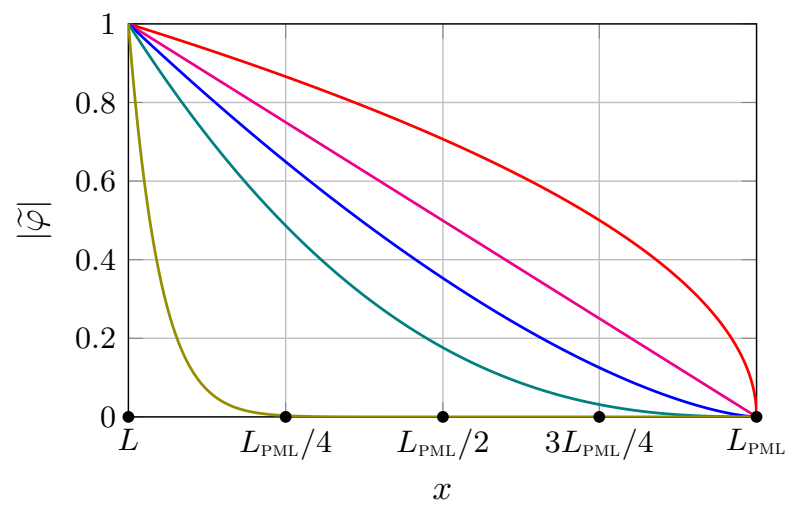
(a) $\sigma_{0}=V_{\mathrm{p}} / 2(-), \sigma_{0}=V_{\mathrm{p}}(-), \sigma_{0}=3 V_{\mathrm{p}} / 2(-)$ and $\sigma_{0}=20 V_{\mathrm{p}}(-)$
$\sigma_{0}=5 V_{\mathrm{p}} / 2(-$

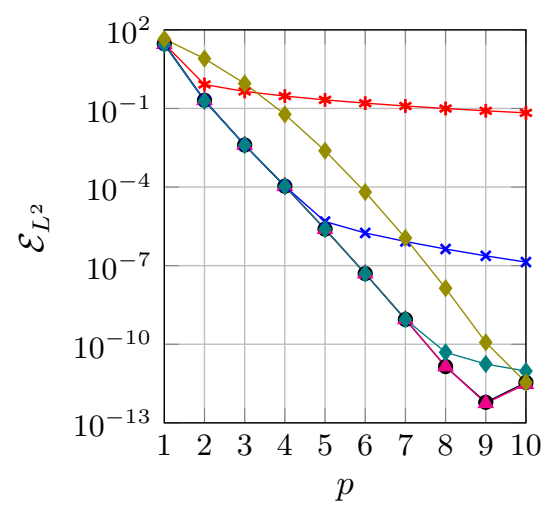

(b) $\sigma_{0}=V_{\mathrm{p}} / 2\left(\multimap-\sigma_{0}=V_{\mathrm{p}}(\multimap), \sigma_{0}=3 V_{\mathrm{p}} / 2(\multimap)\right.$, $\sigma_{0}=5 V_{\mathrm{p}} / 2(\longrightarrow)$ and $\sigma_{0}=20 V_{\mathrm{p}}(\longrightarrow)$. Reference solution $(\multimap-$.

Figure A.19: Modulus of the exact PML solution in one-dimension and relative $L^{2}$-error (in \%) in $\Omega_{\text {phy }}$ as a function of $p$. Parameters: $n=3, M=0, k_{0}=50$.

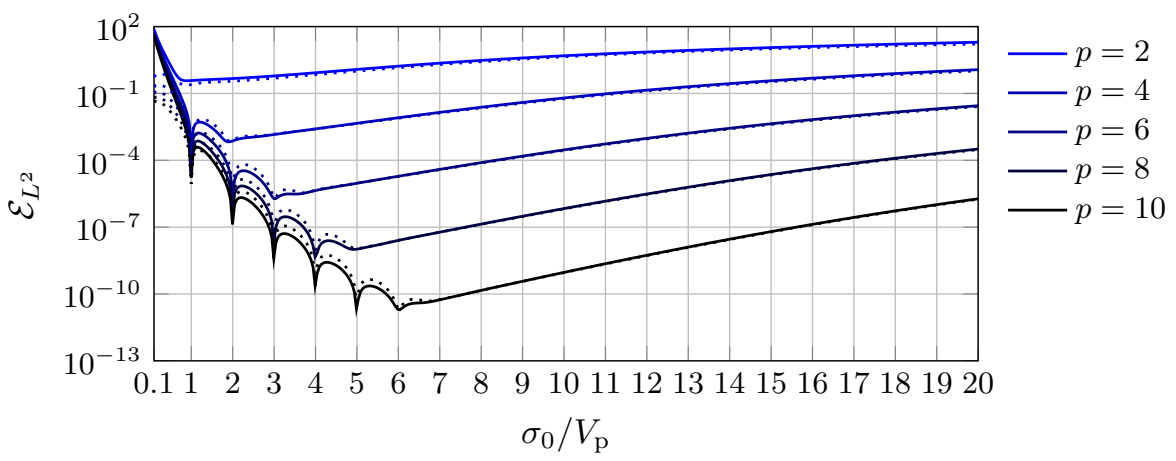

Figure A.20: Relative $L^{2}$-error (-) in $\%$ and corresponding best $L^{2}$-interpolation error $(\cdots \cdots)$ in $\Omega_{\mathrm{PML}}$ for the $2 \mathrm{D}$ duct problem with $k_{0}=50, M=0, n=3$.

The same argumentation holds for the convected problem, the important parameter to consider being the phase velocity seen by the PML. Since the latter clearly depends on the mode, the frequency and the mean flow, we see that there is a priori no perfect choice for $\sigma_{0}$. The criterion $\sigma_{0}>V_{p}$ guarantees a good regularity of the continuous PML solution. This explains the choice in the article, where the value $\sigma_{0}=4 \beta^{2}$ allows to avoid the sub-optimal regime for all PML models in the considered frequency range, thus a fair comparison.

One might extend this analysis to the free field situation. In that case the phase velocity seen by the PML is driven by the incidence angle of the wave, and one would obtain the criterion $\sigma_{0}>c_{0}$ (and $\sigma_{0}>\beta c_{0}$ for the Lorentz model) in order to avoid the sub-optimal regime.

\section{References}

[1] J.-P. Bérenger, A perfectly matched layer for the absorption of electromagnetic waves, Journal of Computational Physics 114 (2) (1994) 185-200.

[2] W. C. Chew, W. H. Weedon, A 3d perfectly matched medium from modified Maxwell's equations with stretched coordinates, Microwave and Optical Technology Letters 7 (13) (1994) 599-604.

[3] F. L. Teixeira, W. C. Chew, Complex space approach to perfectly matched layers: a review and some new developments, International Journal of Numerical Modelling: Electronic Networks, Devices and Fields 13 (5) (2000) 441-455. 
[4] F. Collino, P. Monk, The perfectly matched layer in curvilinear coordinates, SIAM Journal on Scientific Computing 19 (6) (1998) 2061-2090.

[5] F. Collino, P. Monk, Optimizing the perfectly matched layer, Computer Methods in Applied Mechanics and Engineering 164 (1-2) (1998) 157-171.

[6] A. Modave, E. Delhez, C. Geuzaine, Optimizing perfectly matched layers in discrete contexts, International Journal for Numerical Methods in Engineering 99 (6) (2014) 410-437.

[7] A. Bermúdez, L. Hervella-Nieto, A. Prieto, R. Rodríguez, An optimal perfectly matched layer with unbounded absorbing function for time-harmonic acoustic scattering problems, Journal of Computational Physics 223 (2) (2007) 469-488.

[8] A. Bermúdez, L. Hervella-Nieto, A. Prieto, R. Rodríguez, An exact bounded perfectly matched layer for time-harmonic scattering problems, SIAM Journal on Scientific Computing 30 (1) (2008) 312-338.

[9] S. G. Johnson, Notes on Perfectly Matched Layers (PMLs), Lecture Notes, Massachusetts Institute of Technology, https://math.mit.edu/ stevenj/18.369/pml.pdf (2007).

[10] F. Q. Hu, On absorbing boundary conditions for linearized Euler equations by a perfectly matched layer, Journal of Computational Physics 129 (1) (1996) 201-219.

[11] J. S. Hesthaven, On the analysis and construction of perfectly matched layers for the linearized Euler equations, Journal of Computational Physics 142 (1) (1998) 129-147.

[12] S. Abarbanel, D. Gottlieb, J. S. Hesthaven, Well-posed perfectly matched layers for advective acoustics, Journal of Computational Physics 154 (2) (1999) 266-283.

[13] D. Appelö, T. Hagstrom, G. Kreiss, Perfectly matched layers for hyperbolic systems: general formulation, well-posedness, and stability, SIAM Journal on Applied Mathematics 67 (1) (2006) 1-23.

[14] F. Q. Hu, A stable, perfectly matched layer for linearized Euler equations in unsplit physical variables, Journal of Computational Physics 173 (2) (2001) 455-480.

[15] F. Q. Hu, A perfectly matched layer absorbing boundary condition for linearized Euler equations with a non-uniform mean flow, Journal of Computational Physics 208 (2) (2005) 469-492.

[16] E. Bécache, A. S. Bonnet-Bendhia, G. Legendre, Perfectly matched layers for the convected Helmholtz equation, SIAM Journal on Numerical Analysis 42 (1) (2004) 409-433.

[17] T. Hagstrom, A new construction of perfectly matched layers for hyperbolic systems with applications to the linearized Euler equations, in: Mathematical and Numerical Aspects of Wave Propagation WAVES 2003, Springer, 2003, pp. 125-129.

[18] T. Hagstrom, I. Nazarov, Absorbing layers and radiation boundary conditions for jet flow simulations, in: 8th AIAA/CEAS Aeroacoustics Conference \& Exhibit, 2002, p. 2606.

[19] F. Nataf, A new approach to perfectly matched layers for the linearized Euler system, Journal of Computational Physics 214 (2) (2006) 757-772.

[20] F. Dubois, E. Duceau, F. Marechal, I. Terrasse, Lorentz transform and staggered finite differences for advective acoustics, arXiv preprint arXiv:1105.1458.

[21] J. Diaz, P. Joly, A time domain analysis of PML models in acoustics, Computer Methods in Applied Mechanics and Engineering 195 (29-32) (2006) 3820-3853.

[22] E. Bécache, S. Fauqueux, P. Joly, Stability of perfectly matched layers, group velocities and anisotropic waves, Journal of Computational Physics 188 (2) (2003) 399-433.

[23] S. A. Parrish, F. Q. Hu, PML absorbing boundary conditions for the linearized and nonlinear Euler equations in the case of oblique mean flow, International Journal for Numerical Methods in Fluids 60 (5) (2009) 565-589. 
[24] R. J. Astley, Numerical methods for noise propagation in moving flows, with application to turbofan engines, Acoustical Science and Technology 30 (4) (2009) 227-239.

[25] G. Gabard, H. Bériot, A. Prinn, K. Kucukcoskun, Adaptive, high-order finite-element method for convected acoustics, AIAA Journal 56 (8) (2018) 3179-3191.

[26] R. Amiet, W. Sears, The aerodynamic noise of small-perturbation subsonic flows, Journal of Fluid Mechanics 44 (2) (1970) 227-235.

[27] K. Taylor, A transformation of the acoustic equation with implications for wind-tunnel and lowspeed flight tests, Proceedings of the Royal Society of London. A. Mathematical and Physical Sciences 363 (1713) (1978) 271-281.

[28] C. Chapman, Similarity variables for sound radiation in a uniform flow, Journal of Sound and Vibration 233 (1) (2000) 157-164.

[29] J. D. Jackson, Classical electrodynamics, John Wiley \& Sons, 2007.

[30] A. L. Gregory, S. Sinayoko, A. Agarwal, J. Lasenby, An acoustic space-time and the Lorentz transformation in aeroacoustics, International Journal of Aeroacoustics 14 (7) (2015) 977-1003.

[31] A. Gregory, A. Agarwal, J. Lasenby, S. Sinayoko, Geometric algebra and an acoustic space time for propagation in non-uniform flow, arXiv preprint arXiv:1701.04715.

[32] F. Q. Hu, M. E. Pizzo, D. M. Nark, On the use of a Prandtl-Glauert-Lorentz transformation for acoustic scattering by rigid bodies with a uniform flow, Journal of Sound and Vibration 443 (2019) 198-211.

[33] S. Mancini, Boundary integral methods for sound propagation with subsonic potential mean flows, Ph.D. thesis, University of Southampton (2017).

[34] N. Balin, F. Casenave, F. Dubois, E. Duceau, S. Duprey, I. Terrasse, Boundary element and finite element coupling for aeroacoustics simulations, Journal of Computational Physics 294 (2015) 274-296.

[35] M. Visser, Acoustic black holes: horizons, ergospheres and Hawking radiation, Classical and Quantum Gravity 15 (6) (1998) 1767.

[36] W. G. Unruh, Experimental black-hole evaporation?, Physical Review Letters 46 (21) (1981) 1351.

[37] H. Glauert, The effect of compressibility on the lift of an aerofoil, Proceedings of the Royal Society of London. Series A, Containing Papers of a Mathematical and Physical Character 118 (779) (1928) $113-119$.

[38] E. Bécache, P. Joly, M. Kachanovska, V. Vinoles, Perfectly matched layers in negative index metamaterials and plasmas, ESAIM: Proceedings and Surveys 50 (2015) 113-132.

[39] V. Vinoles, Problèmes d'interface en présence de métamatériaux: modélisation, analyse et simulations, Ph.D. thesis, Université Paris-Saclay (2016).

[40] S. W. Rienstra, A. Hirschberg, An Introduction to Acoustics, https://www.win.tue.nl/ sjoerdr/ papers/boek.pdf, extended and revised edition of IWDE 92-06 (2004).

[41] C. Geuzaine, J.-F. Remacle, Gmsh: A 3-D finite element mesh generator with built-in pre-and postprocessing facilities, International Journal for Numerical Methods in Engineering 79 (11) (2009) 13091331.

[42] P. Solin, K. Segeth, I. Dolezel, Higher-order finite element methods, Chapman and Hall/CRC, 2003.

[43] H. Bériot, A. Prinn, G. Gabard, Efficient implementation of high-order finite elements for Helmholtz problems, International Journal for Numerical Methods in Engineering 106 (3) (2016) 213-240.

[44] H. Bériot, G. Gabard, E. Perrey-Debain, Analysis of high-order finite elements for convected wave propagation, International Journal for Numerical Methods in Engineering 96 (11) (2013) 665-688. 
[45] A. Düster, E. Rank, B. Szabó, The p-version of the finite element and finite cell methods, Encyclopedia of Computational Mechanics Second Edition (2017) 1-35.

[46] R. Cimpeanu, A. Martinsson, M. Heil, A parameter-free perfectly matched layer formulation for the finite-element-based solution of the Helmholtz equation, Journal of Computational Physics 296 (2015) $329-347$.

[47] S. Kim, Error analysis of PML-FEM approximations for the Helmholtz equation in waveguides., ESAIM: Mathematical Modelling \& Numerical Analysis 53 (4) (2019) 1191-1222.

[48] A. El Kacimi, O. Laghrouche, D. Ouazar, M. Mohamed, M. Seaid, J. Trevelyan, Enhanced conformal perfectly matched layers for Bernstein-Bézier finite element modelling of short wave scattering, Computer Methods in Applied Mechanics and Engineering 355 (2019) 614-638.

[49] C. Michler, L. Demkowicz, J. Kurtz, D. Pardo, Improving the performance of perfectly matched layers by means of hp-adaptivity, Numerical Methods for Partial Differential Equations: An International Journal 23 (4) (2007) 832-858.

[50] T. Köppl, B. Wohlmuth, Optimal a priori error estimates for an elliptic problem with Dirac right-hand side, SIAM Journal on Numerical Analysis 52 (4) (2014) 1753-1769.

[51] T. Dohnal, Perfectly matched layers for coupled nonlinear Schrödinger equations with mixed derivatives, Journal of Computational Physics 228 (23) (2009) 8752-8765.

[52] H. Bériot, G. Gabard, Anisotropic adaptivity of the p-fem for time-harmonic acoustic wave propagation, Journal of Computational Physics 378 (2019) 234-256.

[53] A. Lieu, G. Gabard, H. Bériot, A comparison of high-order polynomial and wave-based methods for Helmholtz problems, Journal of Computational Physics 321 (2016) 105-125.

[54] O. Ozgun, M. Kuzuoglu, Non-Maxwellian locally-conformal PML absorbers for finite element mesh truncation, IEEE Transactions on Antennas and Propagation 55 (3) (2007) 931-937.

[55] H. Bériot, M. Tournour, On the locally-conformal perfectly matched layer implementation for Helmholtz equation, in: INTER-NOISE and NOISE-CON Congress and Conference Proceedings, no. 8, Institute of Noise Control Engineering, 2009, pp. 503-513.

[56] P. J. Matuszyk, L. F. Demkowicz, Parametric finite elements, exact sequences and perfectly matched layers, Computational Mechanics 51 (1) (2013) 35-45. 